\title{
Marked Differences in the Submandibular Salivary Proteome between Sardinian Alcohol-Preferring and Sardinian Alcohol-Non Preferring Rats Revealed by an Integrated Top-Down-Bottom-Up Proteomic Platform
}

Tiziana Cabras, ${ }^{*}{ }^{\dagger}$ Alfredo D’Alessandro, ${ }^{\dagger}$ Simone Serrao, ${ }^{\dagger}$ Raffaella Isola, ${ }^{\dagger}$ Federica Iavarone, ${ }^{\perp}$ Federica Vincenzoni, ${ }^{\perp}$ Giancarlo Colombo, "Jörgen Ekström, ${ }^{\S} \ddagger$ Irene Messana, ${ }^{\perp, \#}$ and Massimo Castagnola ${ }^{\perp, \#}$

\footnotetext{
${ }^{\dagger}$ Dipartimento di Scienze della Vita e dell’Ambiente and ${ }^{\ddagger}$ Dipartimento di Scienze Biomediche, Università di Cagliari, 09042 Monserrato, Cagliari, Italy

${ }^{\S}$ Department of Pharmacology and Institute of Neuroscience and Physiology, Sahlgrenska Academy at University of Gothenburg, Box 431, SE-405 30 Göteborg, Sweden

"Istituto di Neuroscienze, Sezione di Cagliari, Consiglio Nazionale delle Ricerche (CNR), 09042 Monserrato, Cagliari, Italy

${ }^{\perp}$ Istituto di Biochimica e Biochimica Clinica, Facoltà di Medicina, Università Cattolica, Largo Francesco Vito 1 00168, Rome, Italy

\#Istituto di Chimica del Riconoscimento Molecolare, Consiglio Nazionale delle Ricerche (CNR), Largo Francesco Vito 100168 , Rome, Italy
}

Supporting Information

ABSTRACT: Sardinian alcohol-preferring (sP) and Sardinian alcohol-non preferring (sNP) rats have been selectively bred for opposite alcohol preference and consumption. Aiming to verify possible differences at the proteomics level between sP and sNP rats, we investigated the salivary proteome by a a liquid chromatography-mass spectrometry top-down-bottom-up integrated approach. For this purpose, submandibular saliva was collected from alcohol-naive sP and sNP rats under isoprenaline stimulation. A total of 200 peptides and proteins were detected and quantified in the two rat lines, 149 of which were characterized in their naturally occurring structure. The data are available via ProteomeXchange with identifier PXD006997. Surprisingly, sP rats exhibited marked quantita-

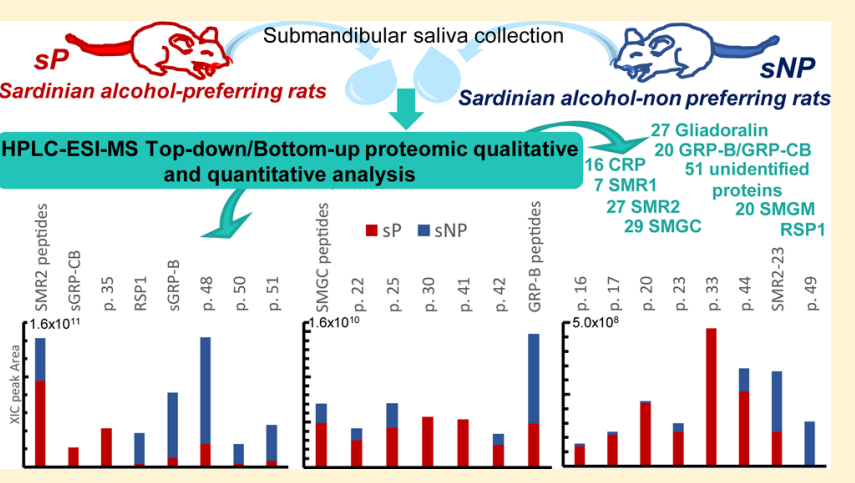
tive and qualitative differences with respect to sNP rats, namely higher levels of proteoforms originating from submandibular gland protein $\mathrm{C}$, and from submandibular rat protein 2 , as well as those of several unidentified peptides and proteins. sP rats expressed some proteins not detectable in sNP rats such as the glutamine and glutamic acid-rich protein (GRP)-CB. The isoform GRP-B, detectable in both rat lines, was more abundant in sNP rats. The submandibular saliva of sNP rats was also characterized by very high levels of GRP-B proteolytic peptides and rat salivary protein 1 . Whether these differences could contribute to the opposite alcohol preference and consumption of sP and sNP rats is currently unknown and requires further investigation.

KEYWORDS: submandibular gland, rat saliva, Sardinian alcohol-preferring rats, Sardinian alcohol-non preferring rats, top-down-bottom-up proteomics, HPLC-ESI-MS, GRPs, RSP1, SMGC, SMR2

\section{INTRODUCTION}

Sardinian alcohol-preferring $(\mathrm{sP})$ rats constitute one of the rat lines selectively bred worldwide for excessive alcohol preference and consumption. When given a choice between $10 \%(\mathrm{v} / \mathrm{v})$ alcohol and water under the standard home cage two-bottle regimen with unlimited access for $24 \mathrm{~h} /$ day, $\mathrm{sP}$ rats display a clear preference for the alcohol solution and consume 6-7 grams of pure alcohol per kilogram of body weight daily. ${ }^{1}$ Voluntary alcohol intake in $\mathrm{sP}$ rats gives rise to relatively high blood alcohol levels and produces measurable psychopharma- cological effects, including the amelioration of genetically based anxiety-related behaviors and locomotor stimulation. ${ }^{1}$ Notably, $\mathrm{sP}$ rats meet all the fundamental requirements posed when defining an animal model of alcoholism, including: (a) oral ingestion of alcohol; (b) attainment of psychopharmacologically relevant blood alcohol levels; (c) willingness to "work" for alcohol (e.g., lever-responding to access alcohol); (d) develop-

Received: September 5, 2017

Published: October 30, 2017 
ment of tolerance to a given effect of alcohol after voluntary alcohol consumption; (e) development of physical or "behavioral" dependence after voluntary alcohol consumption; and (f) occurrence of relapse-like drinking after a period of alcohol abstinence. ${ }^{1,2}$ Conversely, selectively bred Sardinian alcohol-non preferring (sNP) rats avoid alcohol virtually completely, even when exposed for relatively long periods to mixtures containing alcohol and palatable tastants.

Voluntary alcohol consumption in $\mathrm{sP}$ rats is apparently promoted and controlled by psychopharmacological effects rather than by taste perception of alcohol. Indeed, daily alcohol intake in $\mathrm{sP}$ rats remained high and steady irrespective of alcohol concentration, ${ }^{3}$ and the addition of saccharin to the standard alcohol solution did not alter, even minimally, daily alcohol intake in sP rats. ${ }^{4}$ Additionally, sP and sNP did not display any difference in preference for and consumption of sweet-tasting solutions. ${ }^{4}$ However, it cannot be completely ruled out that differences in alcohol taste perception might contribute, at least to some extent, to the opposite alcohol preference and consumption of $\mathrm{sP}$ and $\mathrm{sNP}$ rats. To investigate this issue, our laboratories have recently started a series of experiments aimed at assessing, at both the proteomic and the behavioral (i.e., taste-reactivity test $)^{5}$ level, if and to what extent alcohol taste perception might contribute to differences in alcohol preference and consumption in $\mathrm{sP}$ and sNP rats. This paper intends to be the opening basic study to address the question of the proteomics assessment.

Indeed, taste perception is realized by a complex mechanism involving several oral proteins, ${ }^{6}$ and in humans, the role played by specific parotid salivary proteins in bitter-taste perception has been demonstrated. ${ }^{7}$ Rat salivary proteins with similar function are unknown, but it should be outlined that the rat salivary proteome is largely uncharacterized. The few studies up until now highlighted great differences between rat and human salivary proteome. $^{8-11}$

With the aim to highlight possible significant qualitative and quantitative variations at the proteomics level between $\mathrm{sP}$ and sNP rats, we investigated the protein composition of the submandibular saliva, secreted in response to the intravenous infusion of the $\beta$-adrenoceptor agonist isoprenaline, by applying an integrated reverse-phase high-performance liquid chromatography-electrospray ionization-mass spectrometry (RPHPLC-ESI-MS) top-down and bottom-up platform.

\section{EXPERIMENTAL PROCEDURES}

\section{Chemicals}

All chemicals and reagents were of analytical grade. They were purchased from Sigma-Aldrich (Saint Louis, MI) and Merck (Damstadt, Germany).

\section{Animals and Sample Collection}

The experimental procedures employed in the present study fully complied with European Directive no. 2010/63/EU and the subsequent Italian Legislative Decree no. 26 (March 4, 2014) on the so-called "Protection of Animals Used for Scientific Purposes". A total of 16 male sP and 14 male sNP rats from the 85th generation were used. The choice of using male rats was dictated by the fact that virtually all studies conducted to date to characterize alcohol drinking behavior and pharmacological consequences of alcohol drinking in sP rats used male subjects. ${ }^{1-4}$ Rats were housed three per cage in standard plastic cages with wood chip bedding. The animal facility was under a $12 \mathrm{~h}: 12 \mathrm{~h}$ light/dark cycle (lights on at 7:00 am), at a constant temperature of $22 \pm 2{ }^{\circ} \mathrm{C}$ and relative humidity of approximately $60 \%$. Standard rat chow (Mucedola, Settimo Milanese, Italy) was always available except for the 15 $\mathrm{h}$ preceding the experiment. Tap water was always available. At the time of the experiments, $\mathrm{sP}$ and sNP rats were alcoholnaive. $\mathrm{sP}$ and sNP rats weighed $435 \pm 15$ and $294 \pm 6 \mathrm{~g}$ (mean \pm SEM), respectively; this difference is in line with the commonly monitored, higher body weight of age-matched sP than sNP rats (Colombo et al., unpublished data). The rats were anesthetized with an intraperitoneal dose ( 0.5 milliliters per 100 grams of body weight) of Equitesin (a fresh mixture of $4.25 \%$ chloral hydrate, $2.1 \%$ magnesium sulfate, and $0.97 \%$ pentobarbital sodium). Body temperature was measured with a rectal probe and maintained at $38{ }^{\circ} \mathrm{C}$ by means of a thermostatically controlled blanket. A polyethylene catheter was inserted into the femoral vein to serve as a conduit for the drug infusion, and a tracheal cannula was fitted. The submandibular duct was approached externally and exposed under the mylohyoid muscle. A fine polyethylene tube (filled with distilled water) was inserted into the duct and secured with two ligatures; there was no ongoing secretion of saliva from the glands. In six sP rats and in seven sNP rats, the submandibular duct on both sides were cannulated. In the other rats, the nonduct cannulated gland was removed for morphological purposes and the isolation of secretory granules (findings not presented in this report). Bleeding, if any, was stopped by Spongostan, and the wound was sutured. Isoprenaline hydrochloride (Sigma Chemicals, St. Louis, MO) was continuously infused at a rate of 20 micrograms per kilogram of body weight per minute for $40 \mathrm{~min}$. The first drop of saliva falling from the tip of the cannula represented dead space and was discarded. Saliva was directly collected in preweighed, ice-chilled, Eppendorf tubes containing $250 \mu \mathrm{L}$ of $0.1 \%$ 2,2,2-trifluoroacetic acid (TFA) aqueous solution; in the case of both ducts being cannulated, the saliva was collected in the same tube. Each tube was immediately weighed, and appropriate volumes of $0.1 \%$ TFA were added to obtain the same ratio between saliva and 0.1\% TFA (30:70) in all of the samples. Saliva and TFA were vigorously mixed and centrifuged at $10000 \mathrm{~g}$ for $7 \mathrm{~min}$ at $4{ }^{\circ} \mathrm{C}$, and the acidic supernatants separated from the precipitate and stored at $-80{ }^{\circ} \mathrm{C}$ until RPHPLC-ESI-MS analysis. The total protein concentration was determined by the bicinchoninic acid (BCA) assay kit (SigmaAldrich) and used to normalize protein levels. Before the HPLC-ESI-MS analysis, $120 \mu \mathrm{L}$ of each sample were further diluted in a ratio $1: 10$ with $0.1 \%$ TFA, and $100 \mu \mathrm{L}$ were analyzed by HPLC-ESI-low-resolution MS and $20 \mu \mathrm{L}$ by HPLC-ESI-high-resolution MS/MS. The submandibular gland was removed, pressed gently between gauze pads, and weighed. The saliva secreted was expressed in microliters, assuming the density of saliva to be $1.0 \mathrm{~g} / \mathrm{mL}$, and expressed per gland or per 100 milligrams of gland mass. In the case of both glands of the same animal being duct-cannulated, means were calculated and used for the statistical analysis.

\section{HPLC-ESI-Low-Resolution MS Experiments}

The searching and quantification of peptides and proteins were made by RP-HPLC-ESI-low-resolution (1:6000) MS analysis of the acid soluble fraction of SM saliva samples. The measurements were carried out by a Surveyor HPLC system connected to an LCQAdvantage mass spectrometer (ThermoFisher Scientific San Jose, CA). The mass spectrometer was equipped with an ESI source. The chromatographic column 
was a Vydac (Hesperia, CA) C8 column with $5 \mu \mathrm{m}$ particle diameter $(150 \mathrm{~mm} \times 2.1 \mathrm{~mm})$. The following solutions were used: (eluent A) $0.056 \%(\mathrm{v} / \mathrm{v})$ aqueous TFA, and (eluent B) $0.05 \%(\mathrm{v} / \mathrm{v})$ TFA in acetonitrile-water 80:20. The gradient applied for the analysis of saliva was the following: from 0 to $23 \%$ of B in $10 \mathrm{~min}$, from $23 \%$ to $70 \%$ of B in $45 \mathrm{~min}$, and from $70 \%$ to $100 \%$ in $10 \mathrm{~min}$, at a flow rate of $0.10 \mathrm{~mL} / \mathrm{min}$. During the first $5 \mathrm{~min}$ of separation, the eluate was diverted to waste to avoid instrument damage because of the high salt concentration. Mass spectra were collected every $3 \mathrm{~ms}$ in the $\mathrm{m} / z$ range 300-2000 in positive ion mode. The MS spray voltage was $5.0 \mathrm{kV}$, and the capillary temperature was $260{ }^{\circ} \mathrm{C}$. Experimental mass values were obtained by deconvolution of averaged ESI-MS spectra automatically performed using MagTran 1.0 software $^{12}$ and compared with those present in the UniProtKB rat proteome DataBank (http://us.expasy.org/ tools).

\section{Characterization of Salivary Peptides and Proteins}

To characterize peptides and proteins present in SM saliva of rats, we applied a top-down proteomic approach on the intact proteins and peptides present in the acidic supernatant of SM saliva. Enriched HPLC fractions of some proteins were analyzed both by a top-down and a bottom-up approach for their characterization. Enriched fractions were obtained by preparative RP-HPLC (Dionex Ultimate 3000 instrument, ThermoFisher Scientific, Sunnyvale, CA) of SM saliva. The chromatographic column was a reverse-phase Vydac (Hesperia, CA) C8 column with a $5 \mu \mathrm{m}$ particle diameter $(250 \mathrm{~mm} \times 10$ $\mathrm{mm})$. The solutions used for preparative RP-HPLC were the same utilized for analytical HPLC-ESI-MS experiments. The gradient was linear from 0 to $60 \% \mathrm{~B}$ in $40 \mathrm{~min}$ and from 60 to $100 \% \mathrm{~B}$ in $5 \mathrm{~min}$, with a flow rate of $2.8 \mathrm{~mL} / \mathrm{min}$. Fractions corresponding to different peaks were collected separately and lyophilized. Each fraction was solubilized in $100 \mu \mathrm{L}$ of ultrapure $\mathrm{H}_{2} \mathrm{O}$, and $1 / 3$ of the solution was acidified with $0.1 \%$ TFA (1:1 $\mathrm{v} / \mathrm{v}$ ratio) to be checked by low-resolution HPLC-ESI-MS. The protein concentration of each fraction was determined by the BCA assay.

\section{Proteolytic Digestion for the Bottom-Up Analysis}

For proteolytic cleavage, $0.4 \mu \mathrm{g}$ of proteins were mixed with 0.1 $\mu \mathrm{g}$ of trypsin or endoproteinase Glu-C. The tryptic digestion was performed by using the kit "Trypsin Singles Proteomic Grade" (Sigma-Aldrich) according to the manufacturer's instructions. The Glu-C digestion was performed in $100 \mathrm{mM}$ ammonium bicarbonate $(\mathrm{pH} 8.0)$ at $37{ }^{\circ} \mathrm{C}$ overnight. Digestions was stopped by acidification with $0.1 \%$ formic acid (FA) final concentration, and the solutions were stored at -80 ${ }^{\circ} \mathrm{C}$ until the analysis by HPLC-ESI-high-resolution MS/MS.

\section{HPLC-ESI-High-Resolution MS/MS Experiments}

The experiments were carried out by an Ultimate 3000 RSLC Nano System HPLC apparatus (ThermoFisher Scientific, Sunnyvale, CA) coupled to an LTQ Orbitrap Elite apparatus (ThermoFisher Scientific). The columns were a Zorbax 300SBC8 column ( $3.5 \mu \mathrm{m}$ particle diameter; $1.0 \mathrm{~mm} \times 150 \mathrm{~mm})$ for the top-down analysis, and a Zorbax 300 SB-C18 column (3.5 $\mu \mathrm{m}$ particle diameter; $1.0 \mathrm{~mm} \times 150 \mathrm{~mm}$ ) for the bottom-up. Eluents were: (eluent A) $0.1 \%(\mathrm{v} / \mathrm{v})$ aqueous FA, and (eluent B) $0.1 \%(\mathrm{v} / \mathrm{v})$ FA in acetonitrile/water, 80:20. For top-down analyses, the gradient was: $0-2 \mathrm{~min} 5 \% \mathrm{~B}, 2-40 \mathrm{~min}$ from $5 \%$ to $55 \% \mathrm{~B}$ (linear), and $41-43 \mathrm{~min}$ from $55 \%$ to $100 \% \mathrm{~B}$, at a flow rate of $50 \mu \mathrm{L} / \mathrm{min}$. For bottom-up analyses was: $0-2 \mathrm{~min}$
$5 \% \mathrm{~B}, 2-40 \mathrm{~min}$ from $5 \%$ to $70 \% \mathrm{~B}$ (linear), and $41-45 \mathrm{~min}$ from $70 \%$ to $99 \%$ B. MS and MS/MS spectra were collected in positive mode with the resolution of 60000 and 30000, respectively. The $m / z$ range was from 600 to 2000 in the topdown experiments, from 300 to 2000 in the bottom-up experiments. Tuning parameters were as thus: capillary temperature was $250{ }^{\circ} \mathrm{C}$, and the source voltage $4.0 \mathrm{kV}$; capillary voltage and tube lens voltage were 37 and $150 \mathrm{~V}$ in the top-down and 48 and $150 \mathrm{~V}$ in the bottom-up experiments. In data-dependent acquisition mode the three most abundant ions were selected and fragmented by using collision-induced dissociation (CID, 35\% normalized collision energy for 30 $\mathrm{ms}$, with an isolation width of $6-10 \mathrm{~m} / z$, activation $\mathrm{q}$ of 0.25 ). The inject volume was $20 \mu \mathrm{L}$. HPLC-ESI-MS and MS/MS data were generated by Xcalibur 2.2 SP1.48 (Thermo Fisher Scientific) using default parameters of the Xtract program for the deconvolution. MS/MS data were analyzed by the Proteome Discoverer 1.4 program, based on SEQUEST HT cluster as a search engine (University of Washington, licensed to Thermo Electron Corp., San Jose, CA) against the UniProtKB rat proteome (February 2014 released; 33591 entries). For peptide matching, high-confidence filter settings were applied: the peptide score threshold was 2.3; the limits for FDR setting were Xcorr scores greater than 1.2 for singly charged ions and 2.0 and 2.5 for doubly and triply charged ions, respectively. Furthermore, the cleavage specificity was set to trypsin, or endoproteinase GluC, with two missed cleavages in the bottom-up analysis. Precursor mass search tolerance was 10 ppm, and fragment mass tolerance was 0.8 Da. Fixed modifications considered in the bottom-up analysis were carbamidomethyl-Cys. The following variable modifications were searched: phosphorylation, acetylation, pyro-glutamination, and oxidation of methionine residue. De novo sequencing by manual inspection of MS/MS spectra was performed to identify several peptides and proteins with the help of the BLAST program available at https://blast.ncbi.nlm.nih.gov. Peptide sequences and sites of covalent modifications were validated by manual inspection of the experimental fragmentation spectra against the theoretical ones generated by MSProduct software, available at the ProteinProspector Web site (http://prospector.ucsf.edu/prospector/mshome.htm). The mass spectrometry proteomics data have been deposited to the ProteomeXchange Consortium via the $\mathrm{PRIDE}^{13}$ partner repository with the data set identifier PXD006997.

\section{Quantification}

The area of the RP-HPLC-ESI-low-resolution MS extracted ion current (XIC) peaks (signal-to-noise ratio of $>5$ ) was used for quantification. The XIC search reveals the peak associated with the protein of interest by extracting its specific multiply charged ions along the total ion current chromatographic profile. Area of the XIC peaks is proportional to the peptide and protein concentration; therefore, under constant analytical conditions, it can be used for a quantitative analysis and comparative studies. ${ }^{14}$ The estimated percentage error of the XIC procedure was $<8 \%$. A window of $\pm 0.5 \mathrm{Da}$ was used to extract XIC peaks. The multiply charged ions used to selectively extract and quantify the proteins and peptides was carefully selected to exclude values in common with other co-eluting proteins. The XIC peak areas were corrected for the dilution factor and normalized to the total protein concentration. 


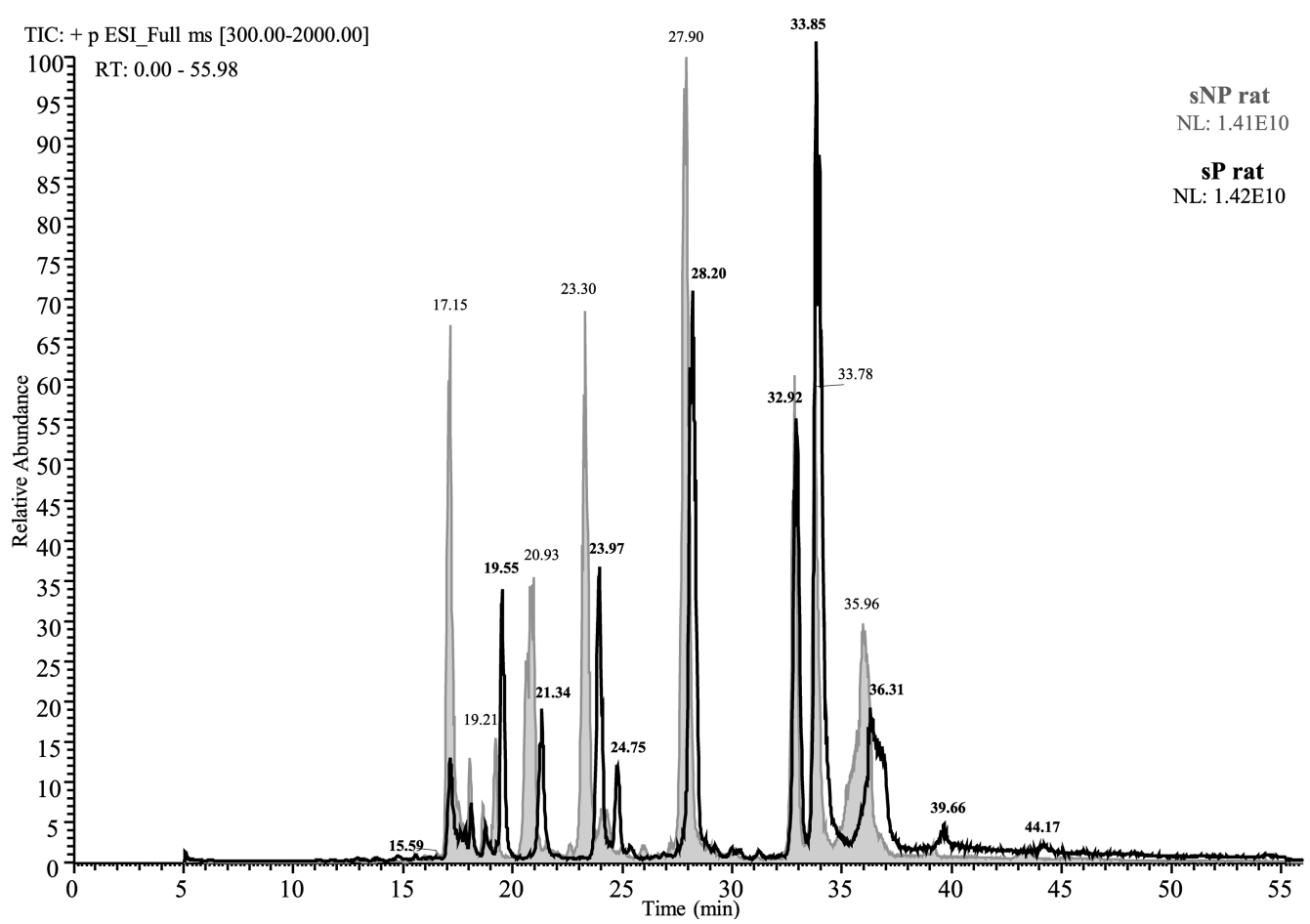

Figure 1. Comparison of the typical HPLC-ESI-low-resolution MS total ion current (TIC) chromatograms of the acidic soluble fraction of SM saliva from sP (black profile) and sNP (gray profile) rats.

\section{Statistical Analysis}

GraphPad Prism (version 5.0) was used for statistical analysis. Means and standard deviations were calculated for XIC peak area of all the peptides and proteins. The following statistical tests were used depending on data distribution (normal or skewed), and variance (homogeneous or unequal): parametric $t$ test (variance homogeneous and normal distribution), $t$ test with Welch correction (normal distribution and variance unequal), and the nonparametric Mann-Whitney test (skewed distribution and variance unequal).

\section{RESULTS}

The glands of the sP rats were heavier, by $21 \%(p<0.001)$, than those of the sNP rats $(295 \pm 10 \mathrm{mg}, n=16$, versus $243 \pm$ $6 \mathrm{mg}, n=14)$. The total volume of submandibular saliva secreted in response to the isoprenaline infusion was larger by $32 \%(p<0.001)$, in the sP rats than in the sNP rats $(126 \pm 7$ $\mu \mathrm{L}, n=16$, versus $95 \pm 3 \mu \mathrm{L}, n=14)$. Expressed per 100 milligrams of gland weight, the mean volume of saliva secreted was $44 \pm 3$ and $39 \pm 1 \mu \mathrm{L}$ in $\mathrm{sP}(n=16)$ and $\mathrm{sNP}(n=14)$ rats, respectively, by the comparison based on gland mass, and no statistical significant difference was attained.

Surprisingly, SM salivary proteins of sP and sNP rats showed great qualitative and quantitative differences, as evidenced by the comparison of the typical total ion current (TIC) chromatograms shown in Figure 1 obtained by HPLC-ESIlow-resolution MS analysis of the acidic soluble fraction of submandibular saliva. The acidic treatment of saliva causes the precipitation of several high-molecular-weight proteins ${ }^{15}$ that were not analyzed in this study. Accordingly, we observed components not heavier than $40 \mathrm{kDa}$. Among the 254 components detected, 54 were not considered for the quantitative and structural analyses being present in less than $40 \%$ of the samples in the two groups. To characterize the 200 peptides and proteins included in the study, samples from 7 rats
(4 sP and $4 \mathrm{sNP}$ ) were separately analyzed by top-down HPLC-ESI-high-resolution MS/MS. This approach allowed the characterization of 149 proteins and peptides that have been grouped into height protein families as shown in Tables 1 and 2, which report monoisotopic mass $(M)$ values (experimental and theoretical) and elution times determined by HPLC-ESI-high-resolution MS; $m / z$ values of the multiply charged ions utilized for MS/MS fragmentation experiments are also indicated. The average mass $\left(M_{\mathrm{av}}\right)$ values obtained by HPLC-ESI-low-resolution MS, as well as the multiply charged ions utilized for their quantification, are reported as Table S-1. The mass values, $M_{\mathrm{av}}$ and Monois., of the 51 uncharacterized components, the elution times, and the multiply charged ions utilized for their quantification, are listed in Table S-2. For the characterization of some components, a bottom-up strategy was applied, and the results obtained are summarized in Table 3. Mass spectrometry proteomics data are accessible, with the code PXD006997, at the ProteomeXchange Consortium. ${ }^{16}$

Top-Down and Bottom-Up Characterization of Peptides and Proteins

Submandibular Gland Protein C Species. Different proteoforms resulting from alternate splicing of the $S m g c$ gene and ranging from 100 to $30 \mathrm{kDa}$ are known for the submandibular gland protein C (SMGC). ${ }^{17}$ A total of 29 peptides corresponding to naturally occurring proteolytic fragments of SMGC were characterized by MS/MS experiments (Table 1, from SMGC-1 to SMGC-29), but the intact proteoform of SMGC was not detected in the samples. The cleavage sites generating the SMGC peptides are indicated on the protein sequence by arrows in Figure S-1 (panel A). The Asn residues at position $164,339,626$, and 638, included in the 29 SMGC peptides, were not glycosylated, and other SMGC peptides containing further potential glycosites were not 
Table 1. Experimental and Theoretical Monoisotopic Mass Values (M, Da), Elution Times (ET), and Multiply-Charged Ions $(m / z$ and Charge) Selected for High-Resolution MS/MS Experiments of Proteins and Peptides Characterized in Rat SM Saliva Exhibiting Significant Quantitative Variations between sP and sNP Rats

\begin{tabular}{|c|c|c|c|}
\hline peptides and proteins (fragment ranges) & $\begin{array}{c}\mathrm{ET} \\
(\mathrm{min})\end{array}$ & $\begin{array}{l}\text { experimental monoisotopic } M \\
\text { (theoretical) (Da) }\end{array}$ & $\begin{array}{l}\text { ions fragmented in high-resolution MS/MS } \\
\text { experiments, } m / z \text { (charge) }\end{array}$ \\
\hline \multicolumn{4}{|c|}{ SMGC species (Q6JHY3) } \\
\hline SMGC-1 $(25-30)$ & 14.68 & $580.29 \pm 0.01(580.286)$ & $581.29(+1)$ \\
\hline SMGC-2 (25-32) & 11.62 & $823.42 \pm 0.02(823.419)$ & $824.42(+1)$ \\
\hline SMGC-3 (33-39) & 31.18 & $717.45 \pm 0.02(717.4429)$ & $718.45(+1)$ \\
\hline SMGC-4 (33-48) & 24.40 & $1617.99 \pm 0.03(1617.982)$ & $540.33(+3)$ \\
\hline SMGC-5 (40-71) & 10.45 & $3191.58 \pm 0.06(3191.577)$ & $639.32(+5), 798.90(+4)$ \\
\hline SMGC-6 (44-71) & 12.07 & $2791.33 \pm 0.06(2791.333)$ & $698.84(+4)$ \\
\hline SMGC-7 (47-71) & 9.87 & $2490.17 \pm 0.05(2490.169)$ & $623.55(+4)$ \\
\hline SMGC-8 (49-71) & 10.65 & $2291.04 \pm 0.04(2291.037)$ & $764.68(+3), 764.68(+3)$ \\
\hline SMGC-9 (59-71) & 10.68 & $1317.63 \pm 0.02(1317.627)$ & $659.82(+2)$ \\
\hline SMGC-10 (104-113) & 23.21 & $1218.63 \pm 0.02(1218.624)$ & $610.32(+2)$ \\
\hline SMGC-11 (104-117) & 34.72 & $1671.94 \pm 0.03(1671.931)$ & $836.97(+2)$ \\
\hline SMGC-12 (118-141) & 14.91 & $2326.11 \pm 0.04(2326.104)$ & $1164.06(+1), 776.37(+3)$ \\
\hline SMGC-13 (142-174) & 16.21 & $3217.46 \pm 0.06(3217.461)$ & $1073.49(+3)$ \\
\hline SMGC-14 (143-151) & 7.46 & $883.41 \pm 0.02(883.404)$ & $884.41(+1)$ \\
\hline SMGC-15 (249-271) & 32.93 & $2545.36 \pm 0.05(2545.350)$ & $637.34(+4)$ \\
\hline SMGC-16 (291-312) & 11.36 & $2087.98 \pm 0.04(2087.983)$ & $697.0(+3)$ \\
\hline SMGC-17 (293-316) & 13.60 & $2229.02 \pm 0.04(2229.014)$ & $1115.51(+2)$ \\
\hline SMGC-18 (331-349) & 13.55 & $1890.89 \pm 0.03(1890.892)$ & $946.45(+2)$ \\
\hline SMGC-19 (476-494) & 25.58 & $2108.08 \pm 0.04(2108.075)$ & $703.70(+3)$ \\
\hline SMGC-20 (476-498) & 35.82 & $2561.38 \pm 0.05(2561.381)$ & $641.35(+4)$ \\
\hline SMGC-21 (476-515) & 33.36 & $4108.12 \pm 0.08(4108.115)$ & $685.69(+6)$ \\
\hline SMGC-22 (516-528) & 15.49 & $1301.67 \pm 0.02(1301.662)$ & $651.84(+2)$ \\
\hline SMGC-23 (518-528) & 16.70 & $1088.54 \pm 0.02(1088.539)$ & $545.27(+2)$ \\
\hline SMGC-24 (520-528) & 17.64 & $875.42 \pm 0.02(875.417)$ & $876.42(+1)$ \\
\hline SMGC-25 (529-555) & 16.68 & $2717.27 \pm 0.06(2717.274)$ & $906.76(+3)$ \\
\hline SMGC-26 (583-606) & 17.44 & $2719.17 \pm 0.06(2719.171)$ & $680.80(+4), 907.40(+3)$ \\
\hline SMGC-27 (621-636) & 6.78 & $1532.69 \pm 0.03(1532.681)$ & $767.35(+2)$ \\
\hline SMGC-28 (637-649) & 23.37 & $1244.57 \pm 0.02(1244.563)$ & $1245.57(+1)$ \\
\hline SMGC-29 (637-659) & 16.79 & $2173.98 \pm 0.04(2173.983)$ & $1087.99(+2)$ \\
\hline \multicolumn{4}{|c|}{ SMR2 species (P18897) } \\
\hline SMR2-1 (17-94 pyro-Glu) & 29.0 & $8595.8 \pm 0.2(8595.795)$ & $1433.64(+2), 1228.98(+7)$ \\
\hline SMR2-2 (17-116 pyro-Glu) & 20.35 & $11370.4 \pm 0.2(11370.33)$ & $1138.64(+10), 1265.04(+9), 1423.04(+8)$ \\
\hline SMR2-3 (44-94) & 17.34 & $5684.6 \pm 0.1(5684.55)$ & $1137.92(+2), 948.43(+6)$ \\
\hline SMR2-4 (95-111) & 6.53 & $2135.18 \pm 0.04(2135.182)$ & $712.73(+3)$ \\
\hline SMR2-5 (95-137) & 29.86 & $5313.82 \pm 0.08(5313.812)$ & $886.64(+6)$ \\
\hline SMR2-6 (98-116) & 15.02 & $2408.29 \pm 0.04(2408.294)$ & $603.08(+4)$ \\
\hline SMR2-7 (108-113) & 6.03 & $755.40 \pm 0.02(755.393)$ & $756.40(+1)$ \\
\hline SMR2-8 $(108-116$ or $107-115)$ & 16.14 & $1187.65 \pm 0.02(1187.641)$ & $594.83(+2), 1188.65(+1)$ \\
\hline SMR2-9 (109-116) & 16.08 & $1058.60 \pm 0.02(1058.598)$ & $530.30(+2)$ \\
\hline SMR2-10 (110-116) & 15.41 & $944.56 \pm 0.02(944.556)$ & $473.28(+2)$ \\
\hline SMR2-11 (111-116) & 16.01 & $831.48 \pm 0.02(831.472)$ & $416.74(+2)$ \\
\hline SMR2-12 (117-123) & 22.60 & $863.47 \pm 0.02(863.465)$ & $864.47(+1)$ \\
\hline SMR2-13 (117-123 pyro-Glu) & 27.76 & $846.45 \pm 0.02(846.435)$ & $847.45(+1)$ \\
\hline SMR-2-14 (117-130) & 32.06 & $1590.83 \pm 0.03(1590.819)$ & $796.42(+2)$ \\
\hline SMR2-15 (117-131) & 32.95 & $1753.88 \pm 0.03(1753.883)$ & $877.95(+2)$ \\
\hline SMR2-13(16 (117-132) & 30.32 & $1909.99 \pm 0.04(1909.984)$ & $955.99(+2), 637.67(+3)$ \\
\hline SMR2-14(17 (117-132 pyro-Glu) & 32.86 & $1892.96 \pm 0.04(1892.954)$ & $947.49(+2)$ \\
\hline SMR2-15(18 (118-123) & 22.88 & $735.41 \pm 0.02(735.407)$ & $736.41(+1)$ \\
\hline SMR2-16(19 (118-132) & 29.72 & $1781.93 \pm 0.04(1781.925)$ & $891.97(+2)$ \\
\hline SMR2-17(20 (119-132) & 28.02 & $1634.86 \pm 0.03(1634.857)$ & $818.44(+2)$ \\
\hline SMR2-21 (120-132) & 27.19 & $1577.84 \pm 0.03(1577.835)$ & $789.92(+2)$ \\
\hline SMR2-22 (122-131) & 29.80 & $1152.56 \pm 0.02(1152.549)$ & $1153.56(+1)$ \\
\hline SMR2-23 (122-132) & 36.55 & $1308.66 \pm 0.02(1308.650)$ & $655.33(+2)$ \\
\hline SMR2-24 (123-132) & 27.07 & $1211.60 \pm 0.02(1211.597)$ & $1212.60(+1)$ \\
\hline SMR2-25 (124-131) & 25.58 & $908.43 \pm 0.02(908.428)$ & $909.43(+1)$ \\
\hline SMR2-26 (124-132) & 21.08 & $1064.54 \pm 0.02(1064.529)$ & $533.27(+2)$ \\
\hline SMR2-27 (132-137) & 18.24 & $803.41 \pm 0.02(803.408)$ & $804.41(+1)$ \\
\hline
\end{tabular}


Table 1. continued

\begin{tabular}{|c|c|c|c|}
\hline peptides and proteins (fragment ranges) & $\begin{array}{c}\text { ET } \\
(\mathrm{min})\end{array}$ & $\begin{array}{l}\text { experimental monoisotopic } M \\
\text { (theoretical) (Da) }\end{array}$ & $\begin{array}{l}\text { ions fragmented in high-resolution MS/MS } \\
\text { experiments, } m / z \text { (charge) }\end{array}$ \\
\hline \multicolumn{4}{|c|}{ RSP1 (Q63557) } \\
\hline 20-90 pyro-Glu, C-terminal amidated (Asn82 $\rightarrow$ Lys, Val62 $\rightarrow$ Gly) & 35.0 & $7103.1 \pm 0.1(7104.072)$ & $1422.22(+5), 1777.78(+4)$ \\
\hline \multicolumn{4}{|c|}{ CRP species (Q63134) } \\
\hline CRP-1 (192-224) & 24.46 & $3730.92 \pm 0.08(3730.907)$ & $747.19(+5)$ \\
\hline CRP-2 (192-234) & 27.63 & $4906.6 \pm 0.1(4906.621)$ & $701.95(+7)$ \\
\hline CRP-3 (219-224) & 24.71 & $713.45 \pm 0.02(713.448)$ & $714.45(+1)$ \\
\hline CRP-4 (219-234) & 26.55 & $1889.17 \pm 0.04(1889.161)$ & $630.73(+3)$ \\
\hline CRP-5 (223-232) & 26.16 & $1168.67 \pm 0.02(1168.660)$ & $585.33(+2)$ \\
\hline CRP-6 (223-234) & 26.65 & $1437.85 \pm 0.02(1437.846)$ & $480.29(+3)$ \\
\hline CRP-7 (224-234) & 26.55 & $1340.80 \pm 0.02(1340.792)$ & $671.40(+2)$ \\
\hline CRP-8 (225-232) & 17.75 & $924.54 \pm 0.02(624.539)$ & $925.54(+1)$ \\
\hline CRP-9 (225-234) & 29.47 & $1193.73 \pm 0.02(1193.724)$ & $398.91(+3)$ \\
\hline CRP-10 (225-239) & 27.48 & $1775.13 \pm 0.04(1775.126)$ & $5952.71(+3)$ \\
\hline CRP-11 (225-244) & 29.54 & $2285.42 \pm 0.04(2285.418)$ & $572.36(+2)$ \\
\hline CRP-12 (226-232) & 18.69 & $811.46 \pm 0.02(811.455)$ & $812.46(+1)$ \\
\hline CRP-13 (226-234) & 28.67 & $1080.65 \pm 0.02(1080.640)$ & $541.33(+2)$ \\
\hline CRP-14 (226-239) & 28.01 & $1662.05 \pm 0.03(1662.042)$ & $555.02(+3)$ \\
\hline CRP- $15(227-234)$ & 18.60 & $1009.61 \pm 0.02(1009.603)$ & $505.81(+2)$ \\
\hline CRP-16 (235-243) & 17.52 & $953.60 \pm 0.02(953.602)$ & $954.60(+1)$ \\
\hline \multicolumn{4}{|c|}{ GRP-B (Q9JLN8); GRP-CB (P08462) } \\
\hline GRP-B/CB-1 (215-224) & 18.38 & $1185.70 \pm 0.02(1185.702)$ & $593.86(+2)$ \\
\hline GRP-B/CB-2 (215-225) & 27.51 & $1332.78 \pm 0.02(1332.771)$ & $667.39(+2)$ \\
\hline GRP-B/CB-3 (215-227) & 30.63 & $1608.92 \pm 0.03(1608.918)$ & $805.46(+2)$ \\
\hline GRP-B-4 (215-246) & 24.28 & $3923.28 \pm 0.08(3923.280)$ & $654.88(+6)$ \\
\hline GRP-B/CB-5 (220-228) & 29.46 & $1229.70 \pm 0.02(1229.696)$ & $615.85(+2)$ \\
\hline GRP-B/CB-6 (221-227) & 36.86 & $945.51 \pm 0.02(945.500)$ & $946.51(+1)$ \\
\hline GRP-B/CB-7 (221-228) & 32.64 & $1101.60 \pm 0.02(1101.601)$ & $551.81(+2)$ \\
\hline GRP-B/CB-8 (222-228) & 11.64 & $988.52 \pm 0.02(988.517)$ & $495.27(+2)$ \\
\hline GRP-B/CB-9 (223-228) & 32.52 & $841.46 \pm 0.02(841.449)$ & $421.83(+2)$ \\
\hline GRP-B/CB-10 (229-234) & 10.40 & $625.40 \pm 0.01(625.391)$ & $626.40(+1)$ \\
\hline GRP-B/CB-11 (229-236) & 15.30 & $928.56 \pm 0.02(928.561)$ & $465.29(+2)$ \\
\hline GRP-B-12 (229-237) & 18.34 & $1025.62 \pm 0.02(1025.613)$ & $513.81(+2)$ \\
\hline GRP-B-13 (229-238 or $230-239)$ & 14.22 & $1181.72 \pm 0.02(1181.715)$ & $591.86(+2)$ \\
\hline GRP-B-14 (230-238) & 14.23 & $1124.70 \pm 0.02(1124.693)$ & $563.35(+2)$ \\
\hline GRP-B-15 (231-238) & 14.68 & $968.60 \pm 0.02(968.592)$ & $485.30(+2)$ \\
\hline GRP-B-16 (239-244) & 11.09 & $785.42 \pm 0.01(785.429)$ & $786.42(+1)$ \\
\hline GRP-B-17 (239-245 or $238-244)$ & 12.12 & $856.47 \pm 0.02(856.467)$ & $857.47(+1)$ \\
\hline GRP-B-18 (239-246 or $238-247)$ & 11.15 & $1012.57 \pm 0.02(1012.568)$ & $507.29(+2)$ \\
\hline sGRP-CB (28-224) & 19.46 & $21307.0 \pm 0.4(21305.3)$ & $\mathrm{nd}^{a}$ \\
\hline sGRP-B (28-244) & 19.00 & $23814.0 \pm 0.4(23810.7)$ & $\mathrm{nd}^{a}$ \\
\hline
\end{tabular}

identified. On the basis of the known SMGC sequences rich in Thr and Ser residues (161 in total and $18 \%$ and $7 \%$ of the amino acid composition, respectively), the NetOGlyc 4.0 server predicted other possible $\mathrm{O}$-glycoforms of SMGC with a score higher than 0.7 that we were not able to detect. However, we cannot exclude the presence of $\mathrm{N}$ - or $\mathrm{O}$ - glycoforms of SMGC (and of other proteins). Indeed, glycosylation is a modification typically characterized by high heterogeneity that generates crowded and dispersed ESI spectra not resolvable by deconvolution.

Submandibular Rat Protein 2 Species. According to UniProtKB data bank, SMR2 (code P18897) undergoes maturation by removal of an 18-residues signal peptide; thus, the monoisotopic mass value expected for the mature protein is $13666.52 \mathrm{Da}$. This mass value has never been detected in our samples. A pair of SMR2 fragments, out of 27 naturally occurring proteolytic fragments of SMR2, named SMR2-1 (17-94) and SMR2-2 (17-116), and carrying at the $\mathrm{N}$ - terminus a Gln residue cyclized to pyro-glutamic acid (pyroGlu), clearly indicated that the maturation of SMR2 implies the removal of a peptide 16 residues long, in disagreement with the UniProtKB data bank. However, we searched the hypothetical entire mature form of SMR2 (17-137 residues) with and without pyro-Glu unsuccessfully. The mass values of the 27 SMR2 naturally occurring fragments are reported in Table 1. The cleavage sites generating the SMR2 peptides are indicated on the protein sequence by arrows in Figure $S-1$ (panel B). SMR2-2, representing the longest SMR2 protein species detectable, was characterized by HPLC-ESI-high-resolution MS/MS of both the entire form and the tryptic peptides obtained by digestion of purified SMR2-2 (Table 3). In addition, we were able to characterize other fragments of SMR2 carrying N-terminal pyro-Glu, namely SMR2-13 (117-123) and SMR2-17 (117-132) (Table 1).

Rat Salivary Protein 1. The peptide with a monoisotopic $M$ value $7103.1 \pm 0.1 \mathrm{Da}\left(M_{\mathrm{av}}=7106.0 \pm 0.9 \mathrm{Da}\right)$, eluting at 
Table 2. Experimental and Theoretical Monoisotopic Mass Values (M, Da), Elution Times (ET), and Multiply-Charged Ions $(\mathrm{m} / \mathrm{z}$ and charge) Selected for High-Resolution MS/MS Experiments of Proteins and Peptides Characterized in Rat SM Saliva Not Exhibiting Quantitative Variations between sP and sNP Rats. Asterisks Indicate Gliadoralin A Species also Characterized in a Previous Study

\begin{tabular}{|c|c|c|c|}
\hline $\begin{array}{l}\text { peptides and proteins } \\
\text { (fragment ranges) }\end{array}$ & $\begin{array}{l}\mathrm{ET} \\
(\mathrm{min})\end{array}$ & $\begin{array}{c}\text { experimental } \\
\text { monoisotopic } \\
M(\text { theoretical }) \\
(\mathrm{Da})\end{array}$ & $\begin{array}{l}\text { ions fragmented in } \\
\text { high-resolution MS/ } \\
\text { MS experiments, } m / z \\
\text { (charge) }\end{array}$ \\
\hline \multicolumn{4}{|c|}{ gliadoralin A species (D3Z9M3) } \\
\hline $\begin{array}{l}\text { A-1 (17-33, pyro- } \\
\text { Glu) }\end{array}$ & 18.51 & $\begin{array}{l}2028.97 \pm 0.04 \\
(2028.961)\end{array}$ & $677.33(+3)$ \\
\hline $\begin{array}{l}\text { A-2 (fr: } 17-52, \\
\text { pyro-Glu) }\end{array}$ & 18.91 & $\begin{array}{r}4011.9 \pm 0.1 \\
(4011.902)\end{array}$ & $1003.98(+4)$ \\
\hline $\begin{array}{l}\text { A-3 (17-83, pyro- } \\
\text { Glu)* }\end{array}$ & 18.96 & $\begin{array}{c}7705.8 \pm 0.2 \\
(7706.76)\end{array}$ & $\underset{(+7)}{1285.30}(+6), 1101.83$ \\
\hline $\begin{array}{l}\text { A-4 }(17-101 \\
\text { pyro-Glu)* }\end{array}$ & 18.61 & $\begin{array}{l}9870.9 \pm 0.2 \\
(9870.94)\end{array}$ & $1097.77(+9)$ \\
\hline A-5 $(17-106)^{*}$ & 18.75 & $\begin{array}{l}10544.2 \pm 0.2 \\
(10544.25)\end{array}$ & ref 9 \\
\hline A-6 $(22-31)$ & 11.62 & $\begin{array}{l}1150.57 \pm 0.02 \\
\quad(1150.56)\end{array}$ & $1151.57(+1)$ \\
\hline A-7 (22-33) & 17.04 & $\begin{array}{l}1435.71 \pm 0.03 \\
(1435.706)\end{array}$ & $718.86(+2)$ \\
\hline A-8 $(22-52)$ & 18.03 & $\begin{array}{l}3418.65 \pm 0.07 \\
\quad(3418.65)\end{array}$ & $1140.06(+3)$ \\
\hline A-9 $(22-83)^{*}$ & 18.17 & $\begin{array}{c}7112.5 \pm 0.2 \\
(7112.503)\end{array}$ & $\begin{array}{l}1186.42(+6), 1017.08 \\
\quad(+7)\end{array}$ \\
\hline A-10 $(22-101)^{*}$ & 18.00 & $\begin{array}{l}9277.6 \pm 0.2 \\
(9277.75)\end{array}$ & ref 9 \\
\hline A-11 $(22-106)^{*}$ & 18.30 & $\begin{array}{l}9950.0 \pm 0.2 \\
(9949.98)\end{array}$ & ref 9 \\
\hline A-12 (24-33) & 16.86 & $\begin{array}{l}1173.62 \pm 0.02 \\
(1173.610)\end{array}$ & $587.81(+2)$ \\
\hline A-13 $(25-31)$ & 17.56 & $\begin{array}{l}789.40 \pm 0.02 \\
(789.398)\end{array}$ & $790.41(+1)$ \\
\hline A-14 (25-33) & 17.18 & $\begin{array}{l}1074.55 \pm 0.02 \\
(1074.542)\end{array}$ & $538.28(+2)$ \\
\hline A-15 $(32-52)$ & 12.78 & $\begin{array}{l}2286.09 \pm 0.04 \\
(2286.095)\end{array}$ & $1144.05(+2)$ \\
\hline A-16 (32-83) & 16.48 & $\begin{array}{r}5980.0 \pm 0.1 \\
(5979.952)\end{array}$ & $\begin{array}{l}1196.99(+5), 997.66 \\
(+6)\end{array}$ \\
\hline A-17 (32-101) & 16.60 & $\begin{array}{r}8145.1 \pm 0.2 \\
(8145.084)\end{array}$ & $1164.59(+7)$ \\
\hline A-18 (34-52) & 13.30 & $\begin{array}{l}2000.95 \pm 0.04 \\
(2000.951)\end{array}$ & $1001.48(+2)$ \\
\hline A-19 (34-83) & 16.84 & $\begin{array}{r}5694.8 \pm 0.1 \\
(5394.808)\end{array}$ & $\begin{array}{l}1139.97(+5), 950.14 \\
\quad(+6)\end{array}$ \\
\hline A-20 (53-83) & 15.92 & $\begin{array}{l}3711.86 \pm 0.08 \\
(3711.868)\end{array}$ & $\begin{array}{l}1238.29(+3), 928.97 \\
(+4)\end{array}$ \\
\hline A-21 (53-101) & 15.70 & $\begin{array}{r}5877.0 \pm 0.1 \\
(5876.999)\end{array}$ & $980.51(+6)$ \\
\hline A-22 (68-83) & 15.07 & $\begin{array}{l}1900.07 \pm 0.04 \\
(1900.064)\end{array}$ & $951.04(+2)$ \\
\hline A-23 (84-101) & 11.28 & $\begin{array}{l}2183.14 \pm 0.04 \\
(2183.142)\end{array}$ & $728.72(+3)$ \\
\hline A-24 (86-101) & 10.62 & $\begin{array}{l}1998.06 \pm 0.04 \\
(1998.062)\end{array}$ & $667.03(+3)$ \\
\hline A-25 (87-101) & 10.88 & $\begin{array}{l}1901.01 \pm 0.04 \\
(1901.009)\end{array}$ & $634.67(+3)$ \\
\hline A-26 (88-101) & 10.95 & $\begin{array}{l}1787.93 \pm 0.04 \\
(1787.925)\end{array}$ & $\begin{array}{l}894.97(+2), 596.98 \\
(+3)\end{array}$ \\
\hline A-27 (89-101) & 10.97 & $\begin{array}{l}1690.87 \pm 0.04 \\
(1690.872)\end{array}$ & $846.44(+2)$ \\
\hline
\end{tabular}

\begin{tabular}{|c|c|c|c|}
\hline $\begin{array}{l}\text { peptides and proteins } \\
\text { (fragment ranges) }\end{array}$ & $\begin{array}{c}\mathrm{ET} \\
(\mathrm{min})\end{array}$ & $\begin{array}{l}\text { experimental } \\
\text { monoisotopic } \\
M(\text { theoretical }) \\
\text { (Da) }\end{array}$ & $\begin{array}{c}\text { ions fragmented in } \\
\text { high-resolution MS/ } \\
\text { MS experiments, } m / z \\
\text { (charge) }\end{array}$ \\
\hline \multicolumn{4}{|c|}{ SMGM species (Q63549, E9PTY1) } \\
\hline SMGM-1 (23-34) & 8.58 & $\begin{array}{l}1570.79 \pm 0.03 \\
(1570.791)\end{array}$ & $524.60(+3)$ \\
\hline SMGM-2 (35-40) & 17.47 & $\begin{array}{l}760.42 \pm 0.02 \\
(760.412)\end{array}$ & $761.42(+1)$ \\
\hline SMGM-3 (35-41) & 14.04 & $\begin{array}{l}897.48 \pm 0.02 \\
\quad(897.471)\end{array}$ & $898.48(+1)$ \\
\hline SMGM-4 (35-42) & 13.47 & $\begin{array}{c}1053.58 \pm 0.02 \\
(1053.572)\end{array}$ & $527.79(+2)$ \\
\hline SMGM-5 (35-43) & 11.65 & $\begin{array}{l}1209.68 \pm 0.02 \\
(1209.673)\end{array}$ & $605.84(+2)$ \\
\hline SMGM-6 (37-42) & 12.80 & $\begin{array}{l}812.43 \pm 0.02 \\
\quad(812.429)\end{array}$ & $813.43(+1)$ \\
\hline SMGM-7 (43-53) & 12.10 & $\begin{array}{c}1328.70 \pm 0.02 \\
(1328.695)\end{array}$ & $\begin{array}{c}665.35(+2), 443.90 \\
(+3)\end{array}$ \\
\hline SMGM-8 (44-53) & 13.71 & $\begin{array}{l}1172.60 \pm 0.02 \\
(1172.594)\end{array}$ & $\begin{array}{c}1173.60(+1), 587.30 \\
(+2)\end{array}$ \\
\hline SMGM-9 (45-53) & 14.05 & $\begin{array}{c}1035.54 \pm 0.02 \\
(1035.535)\end{array}$ & $1035.54(+1)$ \\
\hline SMGM-10 (48-53) & 11.68 & $\begin{array}{l}721.38 \pm 0.02 \\
(721.376)\end{array}$ & $722.38(+1)$ \\
\hline SMGM-11 (54-59) & 10.22 & $\begin{array}{l}782.38 \pm 0.02 \\
(782.371)\end{array}$ & $392.19(+2)$ \\
\hline SMGM-12 (60-66) & 15.40 & $\begin{array}{l}933.46 \pm 0.02 \\
\quad(933.460)\end{array}$ & $934.46(+1)$ \\
\hline SMGM-13 (60-71) & 14.10 & $\begin{array}{l}1516.77 \pm 0.03 \\
(1516.767)\end{array}$ & $759.39(+2)$ \\
\hline SMGM-14 (60-82) & 28.08 & $\begin{array}{l}2818.49 \pm 0.06 \\
(2818.481)\end{array}$ & $940.50(+3)$ \\
\hline SMGM-15 (60-83) & 26.27 & $\begin{array}{l}2974.59 \pm 0.06 \\
(2974.584)\end{array}$ & $\begin{array}{c}992.53(+3), 744.65 \\
(+4)\end{array}$ \\
\hline SMGM-16 (67-82) & 26.37 & $\begin{array}{l}1903.04 \pm 0.04 \\
(1903.032)\end{array}$ & $952.52(+2)$ \\
\hline SMGM-17 (67-83) & 25.19 & $\begin{array}{l}2059.14 \pm 0.04 \\
(2059.133)\end{array}$ & $\begin{array}{c}1030.57(+2), 687.30 \\
(+3)\end{array}$ \\
\hline SMGM-18 (76-83) & 24.89 & $\begin{array}{l}1024.55 \pm 0.02 \\
(1024.545)\end{array}$ & $1025.55(+1)$ \\
\hline SMGM-19 (93-100) & 12.80 & $\begin{array}{l}1087.56 \pm 0.02 \\
(1087.557)\end{array}$ & $544.78(+2)$ \\
\hline SMGM-20 (94-100) & 9.08 & $\begin{array}{l}974.48 \pm 0.02 \\
\quad(974.472)\end{array}$ & $488.24(+2)$ \\
\hline \multicolumn{4}{|c|}{ SMR1 species (P13432, G3V947) } \\
\hline SMR1-1 $(28-70)$ & 28.98 & $\begin{array}{r}4818.6 \pm 0.1 \\
(4818.563)\end{array}$ & $\underset{(+6)}{964.72}(+5), 804.10$ \\
\hline SMR1-2 (29-70) & 29.54 & $\begin{array}{r}4663.5 \pm 0.1 \\
(4663.452)\end{array}$ & $933.50(+5)$ \\
\hline SMR1-3 (34-70) & 30.22 & $\begin{array}{r}4030.2 \pm 0.1 \\
(4030.139)\end{array}$ & $1008.54(+4)$ \\
\hline SMR1-4 (35-70) & 30.85 & $\begin{array}{l}3874.08 \pm 0.08 \\
\quad(3874.038)\end{array}$ & $969.52(+4)$ \\
\hline SMR1-5 (63-70) & 30.37 & $\begin{array}{l}933.55 \pm 0.02 \\
\quad(933.540)\end{array}$ & $934.55(+1)$ \\
\hline SMR1-6 (71-92) & 33.39 & $\begin{array}{l}2313.27 \pm 0.05 \\
(2313.262)\end{array}$ & $\begin{array}{c}1157.64(+2), 772.09 \\
(+3)\end{array}$ \\
\hline SMR1-7 (71-95) & 32.88 & $\begin{array}{l}2732.45 \pm 0.05 \\
(2732.442)\end{array}$ & $911.82(+3)$ \\
\hline
\end{tabular}

$35.0 \mathrm{~min}$, was identified as rat salivary protein 1 (RSP1) (Table 1 ), even if the sequence determined showed some discordance with that one reported in UniProtKB data bank (code Q63557). The peptide was de novo sequenced by manual inspection of high-resolution MS/MS spectra obtained on the entire form and on the proteolytic peptides derived from trypsin and endoproteinase Glu-C digestion of an enriched HPLC fraction (Table 3). The MS/MS analysis on the $[\mathrm{M}+$ $5 \mathrm{H}]^{5+}$ ion at $1422.22 \mathrm{~m} / z$ corresponding to the intact peptide provided a partial sequence that showed $100 \%$ identity with the 
Table 3. Results of the High-Resolution HPLC-ESI-MS/MS Analysis of the Proteolytic Peptides Obtained by Digestion with Endo-Proteinase Glu-C and Trypsin of Enriched Fractions of SMR2-2, RSP1, sGRP-B, and sGRP-CB ${ }^{a}$

\begin{tabular}{|c|c|c|}
\hline proteolytic peptide sequence and position (fragment ranges) & $\begin{array}{c}\text { exp monoisotopic } \\
\text { (theoretical) }[\mathrm{M}+\mathrm{H}]^{+} \\
m / z \text { values ET }(\mathrm{min})\end{array}$ & $\begin{array}{c}\text { MS/MS analysis } m / z \text { and } \\
\text { charge }\end{array}$ \\
\hline \multicolumn{3}{|c|}{ SMR2-2 (17-116 of SMR2), endoproteinase Glu-C peptides } \\
\hline ISDVKQQPD (28-36) & $\begin{array}{l}1029.53 \pm 0.02 \\
\quad(1029.521)(11.03)\end{array}$ & $515.27(+2)$ \\
\hline ISDVKQQPDSD (28-38) & $\begin{array}{l}1231.58 \pm 0.02 \\
\quad(1231.580)(13.11)\end{array}$ & $616.29(+2)$ \\
\hline VNPGNVQDHE (46-55) & $\begin{array}{l}1108.51 \pm 0.02 \\
\quad(1108.502)(14.16)\end{array}$ & $554.76(+2)$ \\
\hline SAPAANEEPSVSPGNE (56-71) & $\begin{array}{l}1555.69 \pm 0.03 \\
\quad(1555.687)(16.32)\end{array}$ & $778.35(+2)$ \\
\hline PSVSPGNEQEE (64-74) & $\begin{array}{l}1172.51 \pm 0.02 \\
\quad(1172.507)(14.11)\end{array}$ & $586.76(+2)$ \\
\hline QQQQPLPVE (75-83) & $\begin{array}{l}1066.56 \pm 0.02 \\
\quad(1066.553)(23.76)\end{array}$ & $533.78(+2)$ \\
\hline NQEPSDKE (84-91) & $\begin{array}{l}946.41 \pm 0.02(946.411) \\
\quad(11.68)\end{array}$ & $473.71(+2)$ \\
\hline RHRKQKRPPPETLHHRE (92-108) & $\begin{array}{l}2202.23 \pm 0.04 \\
\quad(2202.223)(17.64)\end{array}$ & $551.31(+4)$ \\
\hline TLHHRE (103-108) & $\begin{array}{l}792.41 \pm 0.02(792.396) \\
\quad(12.61)\end{array}$ & $396.71(+2)$ \\
\hline \multicolumn{3}{|l|}{ SMR2-2 (17-116 of SMR2), tryptic peptides } \\
\hline KQKRPPPETLHHR (79-91) & $\begin{array}{l}1623.92 \pm 0.03 \\
\quad(1623.919)(5.17)\end{array}$ & $406.74(+4)$ \\
\hline QKRPPPETLHHR (80-91) & $\begin{array}{l}1495.82 \pm 0.03 \\
\quad(1495.824)(7.27)\end{array}$ & $374.71(+4)$ \\
\hline QKRPPPETLHHRENLRPQIYR (80-100) & $\begin{array}{l}2665.45 \pm 0.05 \\
\quad(2665.455)(16.07)\end{array}$ & $445.08(+6)$ \\
\hline RPPPETLHHR (82-91) & $\begin{array}{l}1239.67 \pm 0.02 \\
\quad(1239.671)(8.48)\end{array}$ & $413.89(+3)$ \\
\hline RPPPETLHHRENLRPQIYR (82-100) & $\begin{array}{l}2409.30 \pm 0.04 \\
\quad(2409.301)(16.60)\end{array}$ & $482.67(+5)$ \\
\hline PETLHHR (85-91) & $\begin{array}{l}889.46 \pm 0.02(889.464) \\
\quad(4.65)\end{array}$ & $445.24(+2)$ \\
\hline ENLRPQIYR (92-100) & $\begin{array}{l}1188.65 \pm 0.02 \\
\quad(1188.648)(18.07)\end{array}$ & $594.83(+2)$ \\
\hline KQKRPPPETLHHRENLRPQIYR (95-116) & $\begin{array}{l}2793.55 \pm 0.06 \\
\quad(2793.549)(15.75)\end{array}$ & $466.60(+6)$ \\
\hline \multicolumn{3}{|l|}{ RSP1 endoproteinase Glu-C peptides } \\
\hline <QDAGTDTADTSDTADGTTDSGTQADATDGQQDAE ${ }^{b}(20-53)$ & $\begin{array}{l}3315.27 \pm 0.07 \\
\quad(3315.265)(16.15)\end{array}$ & $1658.64(+2), 1106.09(+3)$ \\
\hline SSDGTSDAGDGDAPADQDQEDSALLALVKTLKE ${ }^{c, d}(54-86)$ & $\begin{array}{l}3319.53 \pm 0.07 \\
\quad(3319.529)(29.41)\end{array}$ & $1107.18(+3)$ \\
\hline SALLALVKTLKE $^{c}(75-86)$ & $\begin{array}{l}1285.81 \pm 0.02 \\
\quad(1285.809)(27.40)\end{array}$ & $643.41(+2)$ \\
\hline \multicolumn{3}{|l|}{$\begin{array}{ll}\text { RSP1 tryptic peptides }\end{array}$} \\
\hline $\begin{array}{l}<\text { QDAGTDTADTSDTADGTTDSGTQADATDGQQDAESSDGTSDAGDGDAPADQDQEDSALLALVK } \underline{K}^{c, d} \\
(20-82)\end{array}$ & $\begin{array}{l}6144.6 \pm 0.1(6144.507) \\
\quad(26.47)\end{array}$ & $1230.31(+5)$ \\
\hline \multicolumn{3}{|l|}{ sGRP-B endoproteinase Glu-C peptides } \\
\hline TSDVPADSE $(28-36)$ & $\begin{array}{l}920.39 \pm 0.02(920.384) \\
\quad(13.08)\end{array}$ & $460.70(+2)$ \\
\hline TSDVPADSEQQPVDSGSDPPSADADAE (28-54) & $\begin{array}{l}2687.11 \pm 0.05 \\
\quad(2687.108)(18.02)\end{array}$ & $1344.56(+2)$ \\
\hline TSDVPADSEQQPVDSGSDPP SADADAENVQE (28-58) & $\begin{array}{l}3157.32 \pm 0.06 \\
\quad(3157.320)(18.55)\end{array}$ & $1053.45(+3)$ \\
\hline QQPVDSGSDPPSADADAE (37-54) & $\begin{array}{l}1785.75 \pm 0.04 \\
\quad(1785.741)(16.22)\end{array}$ & $893.37(+2)$ \\
\hline QQPVDSGSDPPSADADAENVQE (37-58) & $\begin{array}{l}2255.96 \pm 0.04 \\
\quad(2255.953)(17.96)\end{array}$ & $1128.82(+2)$ \\
\hline STQAENQEPPATSGSEEE (84-101; 107-124) & $\begin{array}{l}1890.79 \pm 0.04 \\
\quad(1890.784)(14.89)\end{array}$ & $946.39(+2)$ \\
\hline NQEPPATSGSE (89-99; 112-122; 135-145) & $\begin{array}{l}1116.48 \pm 0.02 \\
\quad(1116.480)(12.11)\end{array}$ & $558.74(+2)$ \\
\hline NQEPPATSGSEE $(89-100 ; 112-123 ; 135-146)$ & $\begin{array}{l}1245.53 \pm 0.02 \\
\quad(1245.523)(12.77)\end{array}$ & $623.27(+2)$ \\
\hline NQEPPATSGSEEEQQQQE (89-106; 135-152) & $\begin{array}{l}2015.84 \pm 0.04 \\
\quad(2015.843)(14.73)\end{array}$ & $1008.42(+2)$ \\
\hline EQQQQEPTQAE (124-134) & $\begin{array}{l}1315.58 \pm 0.02 \\
\quad(1315.576)(12.01)\end{array}$ & $658.29(+2)$ \\
\hline
\end{tabular}


Table 3. continued

\begin{tabular}{|c|c|c|}
\hline proteolytic peptide sequence and position (fragment ranges) & $\begin{array}{c}\text { exp monoisotopic } \\
{\text { (theoretical) }[\mathrm{M}+\mathrm{H}]^{+}}_{m / z \text { values ET }(\mathrm{min})}\end{array}$ & $\begin{array}{l}\text { MS/MS analysis } m / z \text { and } \\
\text { charge }\end{array}$ \\
\hline \multicolumn{3}{|c|}{ sGRP-B endoproteinase Glu-C peptides } \\
\hline QQQQEPTQAE (125-134) & $\begin{array}{l}1186.53 \pm 0.02 \\
\quad(1186.533)(10.47)\end{array}$ & $593.77(+2)$ \\
\hline STQAENQEPPATSGSEE (107-123) & $\begin{array}{l}1761.74 \pm 0.04 \\
\quad(1761.741)(14.68)\end{array}$ & $881.37(+2)$ \\
\hline EGNVESPPSTPE (175-186) & $\begin{array}{l}1242.55 \pm 0.02 \\
\quad(1242.549)(16.53)\end{array}$ & $621.73(+2)$ \\
\hline NSQEQPQQTNPE (187-198) & $\begin{array}{l}1399.61 \pm 0.03 \\
\quad(1399.608)(12.84)\end{array}$ & $700.31(+2)$ \\
\hline EKPPAPKTQEE (199-209) & $\begin{array}{l}1253.64 \pm 0.02 \\
\quad(1253.637)(9.60)\end{array}$ & $627.32(+2)$ \\
\hline \multicolumn{3}{|c|}{ sGRP-B tryptic peptides } \\
\hline IFPFFIYR (221-228) & $\begin{array}{l}1102.61 \pm 0.02 \\
\quad(1102.608)(31.81)\end{array}$ & $551.81(+2)$ \\
\hline GRPVVVFRPR (229-238) & $\begin{array}{l}1182.72 \pm 0.02 \\
\quad(1182.722)(16.51)\end{array}$ & $394.91(+3)$ \\
\hline GRPVVVFR (229-236) & $\begin{array}{l}929.57 \pm 0.02(929.568) \\
\quad(18.21)\end{array}$ & $465.29(+2)$ \\
\hline \multicolumn{3}{|c|}{ sGRP-CB endoproteinase Glu-C peptides } \\
\hline TSDVPADSE $(28-36)$ & $\begin{array}{l}920.39 \pm 0.02(920.384) \\
\quad(11.78)\end{array}$ & $460.70(+2)$ \\
\hline QQPVDSGSDPPSADADAE (37-54) & $\begin{array}{l}1785.75 \pm 0.04 \\
\quad(1785.741)(14.99)\end{array}$ & $893.37(+2)$ \\
\hline GESAPPANEEPPATSGSEEE (59-78) & $\begin{array}{l}1984.83 \pm 0.04 \\
\quad(1984.825)(16.50)\end{array}$ & $993.42(+2)$ \\
\hline EQQQQEPTQAE (78-88; 101-111; 124-134) & $\begin{array}{l}1315.58 \pm 0.02 \\
\quad(1315.576)(8.98)\end{array}$ & $658.29(+2)$ \\
\hline QQQQEPTQAE (79-88; 102-111; 125-134) & $\begin{array}{l}1186.53 \pm 0.02 \\
\quad(1186.533)(7.11)\end{array}$ & $593.77(+2)$ \\
\hline NQEPPATSGSEE (89-100; 112-123; 135-146) & $\begin{array}{l}1245.53 \pm 0.02 \\
\quad(1245.523)(12.77)\end{array}$ & $623.27(+2)$ \\
\hline NQEPSDSAGEGQE (158-170) & $\begin{array}{l}1347.53 \pm 0.02 \\
\quad(1347.529)(13.23)\end{array}$ & $674.27(+2)$ \\
\hline TQPEEGNVESPPSSPE (171-186) & $\begin{array}{l}1683.74 \pm 0.04 \\
\quad(1683.734)(17.11)\end{array}$ & $842.37(+2)$ \\
\hline EGNVESPPSSPE (175-186) & $\begin{array}{l}1228.53 \pm 0.02 \\
\quad(1228.533)(15.62)\end{array}$ & $614.77(+2)$ \\
\hline GNVESPPSSPE (176-186) & $\begin{array}{l}1099.49 \pm 0.02 \\
\quad(1099.490)(15.47)\end{array}$ & $550.25(+2)$ \\
\hline NSQEQPQQTNPE (187-198) & $\begin{array}{l}1399.61 \pm 0.03 \\
\quad(1399.608)(10.60)\end{array}$ & $700.31(+2)$ \\
\hline EKPPAPKTQEE (199-209) & $\begin{array}{l}1253.64 \pm 0.02 \\
\quad(1253.637)(9.54)\end{array}$ & $627.32(+2)$ \\
\hline
\end{tabular}

${ }^{a}$ Experimental and theoretical monoisotopic $[\mathrm{M}+\mathrm{H}]^{+} \mathrm{m} / z$ values, elution time, sequence, PTMs, and multiply-charged ions selected for MS/MS analysis are reported. ${ }^{b}<\mathrm{Q}: \mathrm{N}$-terminal pyro-Glu. ${ }^{c} \underline{\mathrm{K}}$ : substitution of asparagine for lysine. ${ }^{d} \underline{\mathrm{G}}$ : substitution of valine for glycine.

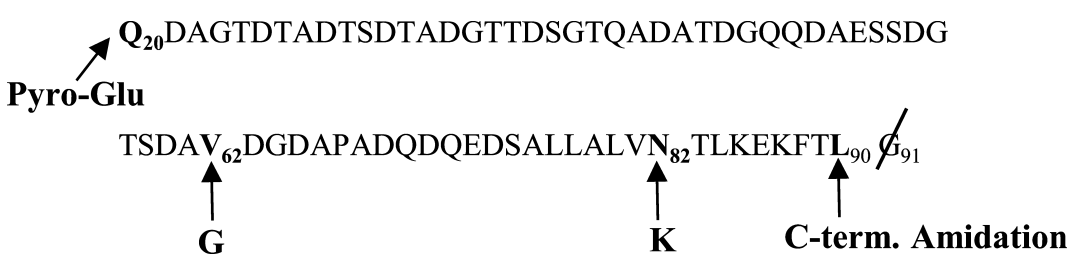

Figure 2. Sequence of RSP1 present in UniProtKB data bank with the entry Q63557. Modifications characterized in this study are indicated by bold characters and arrows: substitutions V62 > G, and N82 > K, N-terminal pyro-glutamination on Q20, C-terminal amidation of L90, and loss of G91. The numbering of the amino acids refers to the polypeptide chain with the signal peptide.

portion 23-47 of RSP1 (http://blast.ncbi.nlm.nih.gov/). The monoisotopic M value of RSP1 corresponds to 7206.1 Da, with $\Delta M$ of $103.0 \pm 0.1 \mathrm{Da}$ with respect to the experimental $M$ value determined by us. However, the analysis of MS/MS spectra of two proteolytic peptides from endoproteinase Glu-C digestion, with experimental $[\mathrm{M}+\mathrm{H}]^{+}$values of $1285.81 \pm 0.02$ and $3319.53 \pm 0.04 \mathrm{~m} / z$, and of the tryptic peptide with experimental $[\mathrm{M}+\mathrm{H}]^{+}$values of $6144.55 \pm 0.08 \mathrm{~m} / z$ (Table
$3)$, evidenced the two substitutions of Asn82 $\rightarrow$ Lys $(\Delta M=$ $+14.1 \mathrm{Da})$ and Val62 $\rightarrow$ Gly $(\Delta M=-42.1 \mathrm{Da})$. Furthermore, the cyclization of N-terminal Gln to pyro-Glu $(\Delta M=-17.0$ $\mathrm{Da})$, the deletion of Gly $91(\Delta M=-57.0 \mathrm{Da})$, and C-terminal amidation $(\Delta M=-1.0 \mathrm{Da})$ allowed to obtain a complete agreement of the experimental MS/MS spectrum with that one expected for RSP1 carrying these modifications (Figure 2). 
Table 4. Results of the Statistical Analysis ( $p$ Value) Obtained by Comparing XIC Peak Area Values (mean \pm SD) of SM Salivary Peptides and Proteins Significantly Different in sP with Respect to sNP Rats ${ }^{a}$

\begin{tabular}{|c|c|c|c|}
\hline peptide and protein (fragment ranges) & $\begin{array}{c}\text { sP XIC peak area } \times 10^{8} \text { mean } \pm \\
\text { SD (frequency) }\end{array}$ & $\begin{array}{l}\text { sNP XIC peak area } \times 10^{8} \text { mean } \\
\pm \text { SD (frequency) }\end{array}$ & $p$ value \\
\hline \multicolumn{4}{|c|}{ most abundant in sP rats } \\
\hline SMGC-1 $(25-30)$ & $0.02 \pm 0.02(7 / 16)$ & 0 & NA \\
\hline SMGC-2 (25-32) & $0.3 \pm 0.23(14 / 16)$ & $0.01 \pm 0.05(1 / 14)$ & $<0.0001$ \\
\hline SMGC-6 (44-71) & $41 \pm 29(16 / 16)$ & $19 \pm 16(13 / 14)$ & $<0.01$ \\
\hline SMGC-11 (104-117) & $0.2 \pm 0.3(7 / 16)$ & 0 & NA \\
\hline SMGC-13 (142-174) & $1.1 \pm 0.8(15 / 16)$ & $0.2 \pm 0.4(5 / 14)$ & $<0.0001$ \\
\hline SMGC-15 (249-271) & $109 \pm 65(16 / 16)$ & $33 \pm 28(11 / 14)$ & 0.0004 \\
\hline SMGC-16 (2191-312) & $0.13 \pm 0.08(16 / 16)$ & $0.004 \pm 0.014(1 / 14)$ & $<0.0001$ \\
\hline SMGC-17 (293-316) & $0.4 \pm 0.2(16 / 16)$ & $0.04 \pm 0.1(5 / 14)$ & $<0.0001$ \\
\hline SMGC-18 (331-349) & $2.3 \pm 1.3(16 / 16)$ & $0.1 \pm 0.3(6 / 14)$ & $<0.0001$ \\
\hline SMGC-21 (476-515) & $1.2 \pm 2.1(6 / 16)$ & $0.02 \pm 0.08(1 / 14)$ & 0.04 \\
\hline SMGC-22 (516-528) & $0.3 \pm 0.4(7 / 16)$ & $0.005 \pm 0.016(2 / 14)$ & 0.02 \\
\hline SMGC-25 (529-555) & $0.1 \pm 0.2(7 / 16)$ & 0 & $\mathrm{NA}$ \\
\hline SMGC-27 (621-636) & $0.8 \pm 0.5(16 / 16)$ & $0.02 \pm 0.08(2 / 14)$ & $<0.0001$ \\
\hline SMGC-28 (637-649) & $0.2 \pm 0.1(16 / 16)$ & $0.01 \pm 0.04(1 / 14)$ & $<0.0001$ \\
\hline SMR2-2 (17-116 pyro-Glu) & $795 \pm 420(15 / 16)$ & $379 \pm 272(11 / 14)$ & 0.003 \\
\hline SMR2-5 (95-137) & $2.4 \pm 2.9(10 / 16)$ & $0.6 \pm 0.7(8 / 14)$ & 0.02 \\
\hline SMR2-7 (108-113) & $0.3 \pm 0.2(16 / 16)$ & $0.2 \pm 0.1(12 / 14)$ & 0.04 \\
\hline SMR2-16 (117-132) & $46 \pm 20(16 / 16)$ & $32 \pm 24(14 / 14)$ & 0.04 \\
\hline SMR2-27 (132-137) & $1.9 \pm 1.7(15 / 16)$ & $0.7 \pm 1.2(9 / 14)$ & 0.04 \\
\hline sGRP-CB (28-224) & $214 \pm 134(16 / 16)$ & 0 & NA \\
\hline peptide no. 9 & $0.5 \pm 0.5(15 / 16)$ & $0.03 \pm 0.04(7 / 14)$ & $<0.0001$ \\
\hline peptide no. 12 & $0.3 \pm 0.2(15 / 16)$ & $0.01 \pm 0.03(2 / 14)$ & $<0.0001$ \\
\hline peptide no. 13 & $0.08 \pm 0.04(16 / 16)$ & 0 & NA \\
\hline peptide no. 16 & $0.7 \pm 0.4(16 / 16)$ & $0.08 \pm 0.2(6 / 14)$ & $<0.0001$ \\
\hline peptide no. 17 & $\pm 0.8(16 / 16)$ & $0.1 \pm 0.3(6 / 14)$ & $<0.0001$ \\
\hline peptide no. 18 & $0.3 \pm 0.1(15 / 16)$ & 0 & NA \\
\hline peptide no. 20 & $2.2 \pm 3.1(7 / 16)$ & $0.06 \pm 0.21(1 / 14)$ & 0.02 \\
\hline protein no. 22 & $30 \pm 25(15 / 16)$ & $13 \pm 13(11 / 14)$ & 0.02 \\
\hline protein no. 23 & $\pm 1.1(15 / 16)$ & $0.3 \pm 0.9(3 / 14)$ & 0.001 \\
\hline protein no. 25 & $44 \pm 16(16 / 16)$ & $27 \pm 12(13 / 14)$ & 0.01 \\
\hline protein no. 26 & $22 \pm 11(15 / 16)$ & 0 & NA \\
\hline protein no. 30 (low-MW protein of pair no. 2) & $56 \pm 48(16 / 16)$ & 0 & NA \\
\hline protein no. 33 ((low-MW protein of pair no. 3$)$ & $4.8 \pm 3.6(16 / 16)$ & 0 & NA \\
\hline protein no. 35 (low-MW protein of pair no. 4) & $426 \pm 224(16 / 16)$ & 0 & NA \\
\hline protein no. 41 & $53 \pm 61(13 / 16)$ & 0 & NA \\
\hline protein no. 42 & $25 \pm 18(14 / 16)$ & $12 \pm 10(12 / 14)$ & 0.02 \\
\hline protein no. 44 & $2.6 \pm 2.1(14 / 16)$ & $0.8 \pm 1.3(6 / 14)$ & 0.008 \\
\hline protein no. 46 (low-MW protein of pair no. 5) & $120 \pm 171(13 / 16)$ & 0 & NA \\
\hline \multicolumn{4}{|c|}{ most abundant in sNP rats } \\
\hline SMR2-23 (122-132) & $1.2 \pm 0.8(16 / 16)$ & $2.1 \pm 1.3(14 / 14)$ & 0.03 \\
\hline RSP1 20-90 pyro-Glu, C-terminal ammidated (Asn82 $\rightarrow$ Lys, Val62 $\rightarrow$ Gly) & $31 \pm 19(16 / 16)$ & $346 \pm 171(14 / 14)$ & $<0.0001$ \\
\hline GRP-B-13 (229-238) & $29 \pm 19(16 / 16)$ & $59 \pm 25(14 / 14)$ & 0.002 \\
\hline GRP-B-14 (230-238) & $2.5 \pm 2.4(16 / 16)$ & $6.0 \pm 3.4(14 / 14)$ & 0.004 \\
\hline GRP-B-18 (239-246) & $17 \pm 11(16 / 16)$ & $34 \pm 15(14 / 14)$ & 0.001 \\
\hline sGRP-B $(28-244)$ & $104 \pm 164(6 / 16)$ & $720 \pm 281(14 / 14)$ & $<0.0001$ \\
\hline protein no. 45 (high-MW protein of pair no. 2) & $20 \pm 35(6 / 16)$ & $135 \pm 100(14 / 14)$ & 0.0004 \\
\hline protein no. 47 (high-MW protein of pair no. 3) & $2.5 \pm 3.6(6 / 16)$ & $15 \pm 12(12 / 14)$ & 0.001 \\
\hline protein no. 48 (high-MW protein of pair no. 4) & $256 \pm 377(6 / 16)$ & $1182 \pm 785(13 / 14)$ & $<0.001$ \\
\hline protein no. 49 & $0.05 \pm 0.21(1 / 16)$ & $1.5 \pm 1.0(12 / 14)$ & $<0.0001$ \\
\hline protein no. 50 & $35 \pm 60(5 / 16)$ & $219 \pm 192(10 / 14)$ & 0.006 \\
\hline protein no. 51 (high-MW protein of pair no. 5) & $71 \pm 138(6 / 16)$ & $394 \pm 516(14 / 14)$ & 0.003 \\
\hline
\end{tabular}

\section{Glutamine and Glutamic Acid Rich Proteins. We} characterized 36 peptides belonging to glutamine and glutamic acid rich proteins encoded by the gene $4 q 43$. This locus is named in three diverse ways in UniProtKB data bank: Grpca,
Grpcb, and Prh1. Grpca encodes for the glutamine and glutamic acid rich protein A (246 residues, GRP-A, P08568). A pair of proteins, GRP-B (248 residues, Q9JLN8) and GRP-CB (247 residues, P08462), are attributed to Grpcb. Prh1 encodes for the 
contiguous repeat polypeptide (CRP, 246 residues, Q63134). Another protein, called Prh1 (245 residues, D4A063) and showing $90 \%$ of homology with CRP, is additionally attributed to Prh1, even though its sequence is labeled as "unreviewed" and is derived from an Ensembl automatic analysis pipeline. We were able to identify 16 peptides originated from CRP, named from CRP-1 to CRP-16 in Table 1. Except for CRP-11 and CRP-16, the other 14 peptides are common to Prhl. No proteotypic peptide of protein Prh1 was observed in our samples, suggesting that CRP is the form selectively encoded by Prh1. The CRP sequence exhibits $97.6 \%$ of identity also with GRP-A, mainly in the 1-244 region, which includes five tandem repeats of 23 amino acid residues rich in proline, glutamine, and glutamic acid. A total of three fragments (CRP3, CRP-11, and CRP-16) are common between CRP and GRPA, but no proteotypic peptide of GRP-A was detected. According to the cleavages sites, which are confined to the C-terminal 192-244 region of the protein (Figure S-1C), the naturally occurring peptides were possibly generated by a trypsin-like activity. A XIC search of tryptic fragments deriving from the 1-191 region did not give any results, as well as the search of the entire forms of CRP, Prh1, and GRP-A. However, the results suggest that only CRP, among the three proteins, was expressed in the rats under study. A total of 18 peptides deriving from GRP-B were characterized; among them, 8 are unique peptides, while 10 are common to GRP-CB, but none of the proteotypic peptide of GRP-CB was detected (Table 1). GRP-B and GRP-CB show 97\% identity, and they share $87 \%$ identity with GRP-A and CRP, mainly at the level of the first 212 residues, presenting 5 tandem repeats of 23 residues. Peptides belonging to GRP-B/CB detected in our samples did not share sequences with CRP and GRP-A because they originated from the C-terminal 215-246 region, probably by a trypsin-like activity (Figure S-1D for GRP-B and S-2A for GRP$\mathrm{CB}$ ). Possible naturally occurring fragments originated by cleavages in the $1-214$ region, as well as the entire forms of GRP-B and GRP-CB, were unsuccessfully searched by XIC procedure.

In the HPLC-ESI-low-resolution MS profile of SM saliva from sNP rats we observed between 16.0 and 26.0 min several proteins clustered in pairs with $M_{\mathrm{av}}$ values between $21316 \pm 3$ and $27731 \pm 4 \mathrm{Da}$, each pair showing a mass difference of 2507 $\pm 1 \mathrm{Da}$, suggesting they may be polymorphic isoforms (Tables S-1 and S-2). Differently, in saliva from sP rats only the lowmolecular-weight protein of every pairs was observed. The pair no. 1 , including the two proteins with $M_{\mathrm{av}}$ values of $21316 \pm 3$ and $23824 \pm 3 \mathrm{Da}$ (Table S-1) (monoisotopic $M$ values of $21307.5 \pm 0.4$ and $23814.0 \pm 0.4 \mathrm{Da}$; Table 1 ), was chosen for a deeper structural characterization and identification. The $M_{\mathrm{av}}$ values for pairs nos. 2, 3, 4, and 5 are reported in Table S-2. The two proteins of pair no. 1 were purified and then submitted to proteolytic digestion with trypsin and endoproteinase Glu-C. The analysis of high-resolution MS/MS of the digestion products allowed the identification of the $21316 \pm 3$ Da protein as the fragment 28-224 of GRP-CB and the 23824 \pm 3 Da protein as the fragment 28-244 of GRP-B, named by us short GRP-CB (sGRP-CB), and short GRP-B (sGRP-B), respectively (Table 3 ). The expected mass value for sGRP-CB $\left(M_{\mathrm{av}}, 21318 \mathrm{Da}\right.$; monoisotopic $\left.M, 21305.3 \mathrm{Da}\right)$ matched well with that observed. Furthermore, the analysis of peptides originated by endoproteinase Glu-C digestion of the $21316 \pm 3$ $\mathrm{Da}$ protein allowed us to cover sequence-encompassing residues $28-146$ and the $158-209$ of GRP-CB. Similarly, the expected mass value for sGRP-B $\left(M_{\mathrm{av}}, 23825 \mathrm{Da}\right.$; monoisotopic $M, 23810.7 \mathrm{Da}$ ) corresponded to the experimental one, and proteolytic fragments from endoproteinase Glu-C covered the sequence encompassing residues 28-58, 84-152, and 175209 of GRP-B, while tryptic fragments covered the C-terminal sequence (residues 221-238). The $\Delta M$ value between the proteins of pair no. 1 agrees with the following differences present in sGRP-B with respect to sGRP-CB: (a) deletion of residues 225-244 (2512 Da) and (b) substitutions $\mathrm{S}>\mathrm{T}(184$ position), Q $>$ E (129), P > S (107), and P > S (84), (overall $-5 \mathrm{Da})$.

Gliadoralin A Species. A total of 27 peptides and proteins have been identified as protein species related to gliadoralin $A$ and were named from A-1 to A-27 (Table 2); six of these were already characterized by us in a previous study ${ }^{9}$ and are marked with an asterisk. N-terminal pyro-glutamination was characterized in four gliadoralin species: A-1, A-2, A-3, and A-4. The cleavage sites originating gliadoralin A peptides are highlighted in the Figure S-2B.

Submandibular Gland Mucin Species. We characterized 20 peptides deriving from the naturally occurring fragmentation of submandibular gland mucin (SMGM), a protein of 309 amino acid residues encoded by Proll gene (Table 2 and Figure S-2C). The two entry codes reported in Table 2 refer to SMGM (code Q63549), and to Proll, an isoform that differs from SMGM for a single substitution into the signal peptide (code E9PTY1). We were not able to detect the entire proteoform, probably due to its elevated level of glycosylation in analogy with the mouse apomucin. ${ }^{18}$

Submandibular Rat Protein 1 Species. The submandibular rat protein 1 (SMR1) was undetectable as entire proteoform (expected monoisotopic mass value of 13489.94 $\mathrm{Da})$, in accordance with Rougeot and colleagues, ${ }^{19}$ that suggested a maturation process of SMR1 like that of peptidehormone precursors. A total of 7 naturally occurring peptides, covering the region ranging from 28 to 95 positions, were characterized by us (Table 2 and Figure S-2D). A pair of entry codes are reported in Table 2, and the code G3V947 identifies an unreviewed isoform of SMR1, called submaxillary gland androgen-regulated protein 3B, differing from SMR1 for two amino acidic substitutions at 5 and 14 positions in the signal peptide.

Summary of the Quantitative Proteomic Differences Observed between Submandibular Saliva from sP and snP Rats

Quantitative label-free proteomic analysis was based on the measurements of the area of extracted ion current peaks. Multicharged ions selected for the XIC quantitation are listed in Table S-2. Results of the statistical analysis, the mean area values \pm standard deviation, and the frequencies of peptides and proteins with significantly diverse levels in sP and in sNP rats are reported in Table 4.

The SM saliva of sP rats exhibited high levels of protein species related to SMGC, SMR2, and sGRP-CB. Among the 29 SMGC peptides identified (Table 1), 15 were sporadically observed in the samples and thus not considered for quantitative comparison; the 14 SMGC peptides quantified (SMGC-1, SMGC-2, SMGC-6, SMGC-11, SMGC-13, SMGC15, SMGC-16, SMGC-17, SMGC-18, SMGC-21, SMGC-22, SMGC-25, SMGC-27, and SMGC-28) were significantly more abundant in sP than in sNPs rats (Table 4). A total of 12 SMR2 peptides were considered for the XIC quantitation (Table S-2), 


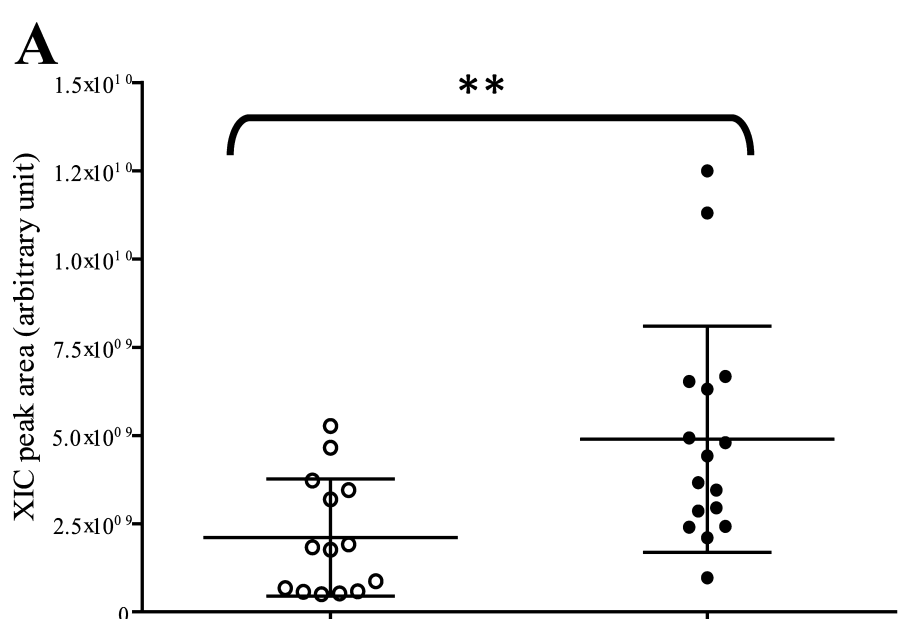

Tot. SMGC sNP
B

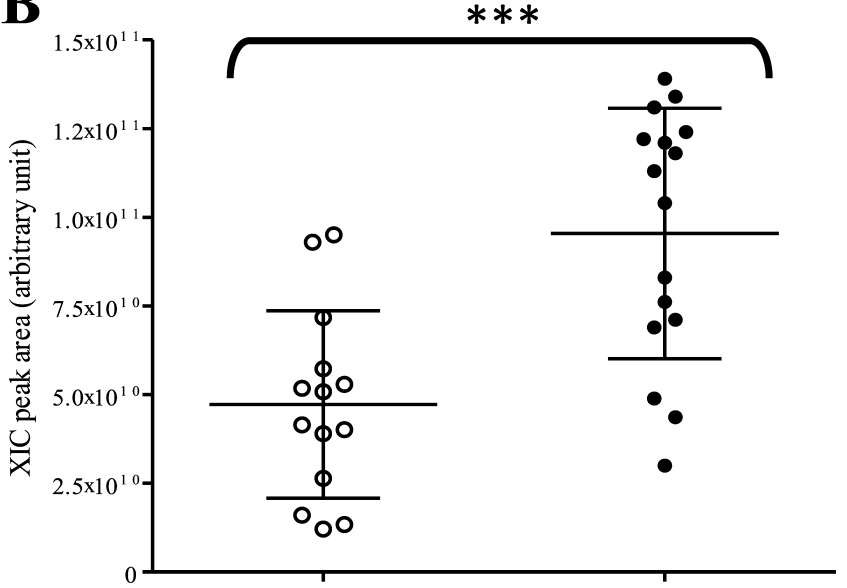

Tot. SMR2 sNP

Tot. SMR2 sP

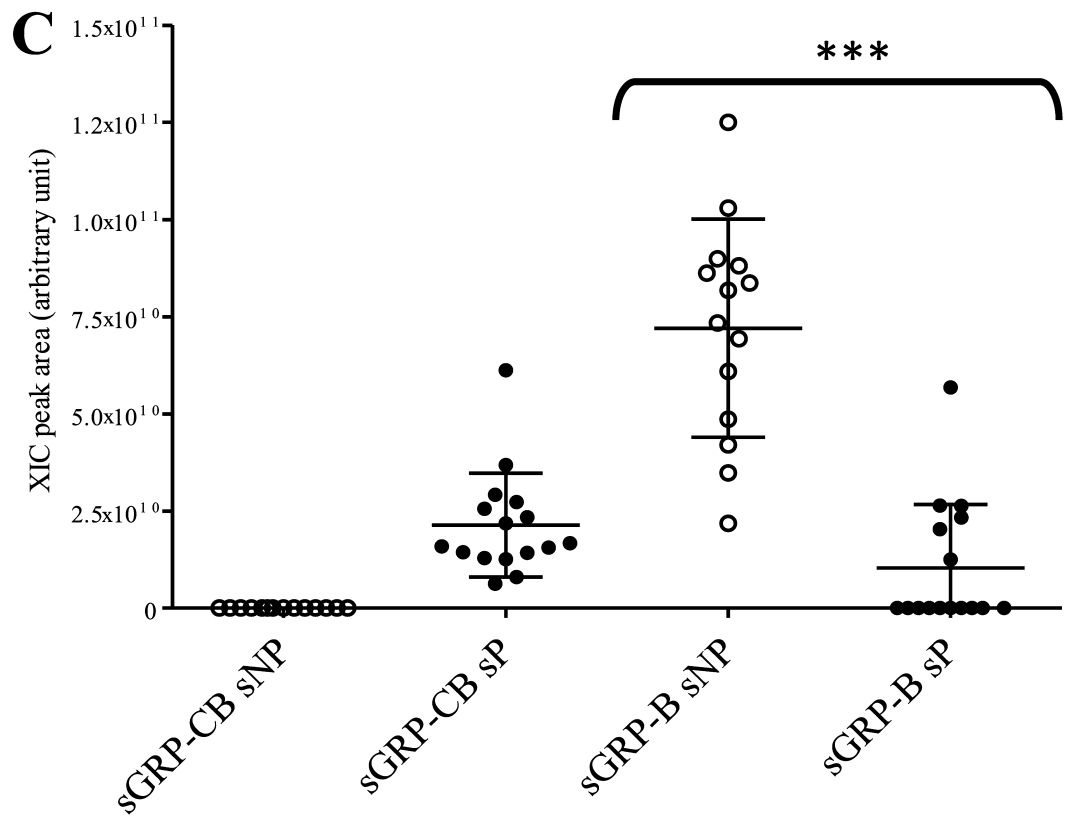

Figure 3. Distribution of the XIC peak areas in sP and sNP rats for the totality of SMGC fragments (A), the totality of SMR2 fragments (B), and the totality of sGRP-CB and sGRP-B (C). Asterisks represent the statistical $p$ values $(*, p<0.05 ; *, p<0.01$; ***, $p<0.001)$.

but only SMR2-2, SMR2-5, SMR2-7, SMR2-16, and SMR2-27 showed a significant higher level in sP rats (Table 4). Figure $3 \mathrm{~A}$ shows the distributions in the two rat groups of the sum of XIC peak areas of SMGC species quantified that one of SMR2 species is shown in Figure 3B. Asterisks represent the statistical $\mathrm{p}$ values $(*, p<0.05$; **, $p<0.01$; ***, $p<$ 0.001 ).

No differences were found in the levels of the peptides that may originate from both GRP-CB and GRP-B (Tables 2, and S2 ); instead sGRP-CB, was detectable only in sP rat saliva (Figure 3C). Analogously, the low-molecular weight proteins of the pairs differing for $2507 \pm 1 \mathrm{Da}$ were detected only in the saliva of sP rats. Several other peptides and proteins that we could not characterize showed higher levels in sP than in sNP rats (Table 4).

The SM saliva of sNP rats showed higher levels of RSP1, SMR2-23, GRP-B-13, GRP-B-14, and GRP-B-18 peptides, sGRP-B, and six uncharacterized proteins, with respect to sP rats (Table 4). Different from sGRP-CB, sGRP-B was expressed in both $\mathrm{sP}$ and $\mathrm{sNP}$ rats, but its level in $\mathrm{sNP}$ rats was 7-fold more abundant (Figure 3C). Analogously, the highmolecular-weight proteins of the pairs differing for $2507 \pm 1 \mathrm{Da}$ were detectable in both rat populations but more abundant in $\mathrm{sNP}$ than in $\mathrm{sP}$ rats.

\section{DISCUSSION}

The key result of this proteomic investigation revealed marked qualitative and quantitative differences in the protein profile of the submandibular saliva between alcohol-naive Sardinian alcohol-preferring rats and Sardinian alcohol-non preferring rats. Saliva secreted at a low continuous rate was evoked from the duct-cannulated submandibular glands in response to the infusion of the $\beta$-adrenoceptor agonist isoprenaline, a saliva known to be relatively rich in proteins. ${ }^{20}$ The total amount of saliva secreted over the $40 \mathrm{~min}$ prolonged period of the isoprenaline infusion, using a low dose of the drug, ${ }^{21}$ was larger (by $32 \%$ ) from the submandibular glands of the sP rats than from those of sNP rats. A difference that seems attributed to the higher gland weights of the sP rats compared those of the sNP rats. 
The great part of the components characterized in the SM secretion were naturally occurring fragments of known proteins, such as gliadoralin, SMGC, SMGM, SMR1, SMR2, RSP1, and glutamine and glutamic acid rich proteins CRP, GRP-B, and GRP-CB. These fragments, being present in SM saliva directly collected from the duct, were generated by proteases, either during the secretory granule maturation or during the transit in the glandular duct. Most fragments originated by trypsin-like cleavages, as suggested by the presence of Lys or Arg residues at the C-terminal position, others by cleavages at Gly, Thr, Ala, Phe, or Val residues, probably by the action of metalloproteinases. SMR2 protein appeared to undergo a complete presecretory proteolysis, and we were able to characterize a family of novel SMR2 fragments covering the entire protein sequence. The fragments of SMR2 protein originating from the $\mathrm{N}$-terminus demonstrated that the signal peptide is 16 and not 18 residues long as reported for UniprotKB data bank entry P18897. Furthermore, these fragments carried an N-terminal pyroglutamic acid moiety, a previously unknown modification for this protein. The $\mathrm{N}$-terminal pyroglutamination is a common post-translational modification of secretory proteins and peptides that is also found in human salivary proteins ${ }^{22}$ and is probably involved in the protection of proteins and peptides from exopeptidase action. ${ }^{23} \mathrm{~N}$-terminal pyroglutamination may be also a requisite for a peptide to assume a conformation appropriate for the receptor interaction. ${ }^{24}$ No information is available on SMR2 function except that the expression of Smr2 in the acini of SM glands is androgen-dependent and that mRNA was found at a higher level (20-30-fold) in male compared to female rat SM glands. ${ }^{25}$ An N-terminal pyroglutamination was first characterized in this study also for RSP1, which carried the substitutions Asn82 $\rightarrow$ Lys and Val62 $\rightarrow$ Gly, lacked the last Gly residue and presented amidation of the C-terminal Leu, with respect to the sequence present in UniProtKB data bank. In this regard, it should be outlined that PTMs of SM rat proteins have been scarcely characterized until now, and sequences present in the data bank were often deduced from cDNA or gene sequences. Differently to the other proteins, we did not detect RSP1 fragments in the SM salivary samples analyzed. The C-terminal amidation appeared an interesting modification, being considered a PTM observed in antimicrobial peptides and useful to cross the plasma membrane. ${ }^{26}$

SMR1, similar to SMR2, was never detected as entire form, probably for two reasons: the presence of glycosyl moieties and the proteolytic processing. Indeed, mRNA sequence analysis predicted that SMR1 would be glycosylated at Asn129 and Asn 136. ${ }^{27}$ SMR1 was defined as a pro-hormone protein generating the bioactive peptides called undecapeptide (fragment 23-33), hexapeptide (fragment 28-33,) and pentapeptide (fragment 29-33). ${ }^{28}$ The pentapeptide is also known as sialorphin, a potent inhibitor of neprilysin with analgesic activity. ${ }^{29}$ More recently, another bioactive SMR1 peptide, the heptapeptide submandibular gland peptide- $T$ (fragment 138144), was studied for its anti-inflammatory property. ${ }^{30,31}$ Although these bioactive peptides are released in response to adrenaline administration in both the saliva and the bloodstreams of adult male rats, ${ }^{28}$ we did not detect them in any of our samples. Whether this difference is attributed to the fact that different strains of rats have been used or that adrenaline not only acts on $\beta$-adrenoceptors, such as isoprenaline, but also acts on $\alpha$-adrenoceptors of the gland ${ }^{32}$ is currently unknown.
A family of novel SMCG naturally occurring fragments were detected and characterized in this study, but, probably due to the reported high-glycosylation status of SMGC, we were unable to detect the entire proteoform in our samples. ${ }^{17,18}$ The biological role of SMGC has not yet been deciphered. The protein is specific marker for type I (terminal tube) cells of neonatal rat SM gland ${ }^{17}$ that are fundamental for SM gland development during the first three postnatal weeks. After the loss of these cells, occurring around 20-30 days after birth, SMGC is expressed by intercalated duct cells. ${ }^{17}$

The glutamine and glutamic acid rich proteins were very hard to characterize due to their high polymorphisms and because the sequences reported in UniprotKB data bank are obtained by genomic and CDNA analysis. ${ }^{33,34}$ The signal peptide in silico predicted for GRP-B and GRP-CB is 16 and 18 amino acid residues long, respectively. The data from our study strongly suggested that the signal peptides of both GRP-B and GRP-CB is 27 residues long. Moreover, both of the fragments sGRP-CB and sGRP-B presented at the C-terminus the sequence ProPhe, suggesting a common proteolytic processing by the action of the same protease during the secretory pathway. A similar cleavage site was recognized in rat angiotensinogen by kallikrein 2 action, an enzyme expressed also in SM glands. ${ }^{35,36}$ The numerous naturally occurring fragments of GRPs characterized in this study confirmed the expression of CRP, GRP-B, and GRP-CB

The detection of other four pairs of proteins with the same $\Delta M$ value $(2507 \pm 1 \mathrm{Da})$ of the pair sGRP-B and sGRP-CB strongly suggested that other GRP isoforms exist. It was not possible to collect structural information on the four protein pairs, and their mass values did not correspond to fragments of known GRPs. We hypothesized they could correspond to correlated protein species, encoded by unknown allelic forms of the $4 \mathrm{q} 43$ locus and carrying structural differences similar to those existing between sGRP-CB and sGRP-B. This hypothesis is reinforced by the fact that all the five protein pairs showed the same quantitative variations in the two rat groups, being the low-molecular-weight protein species of each pair detected only in $\mathrm{sP}$ saliva samples and the high-molecular-weight protein species of each pair detected in both rat groups but with a significantly higher level in saliva from sNP than sP rats.

Other significant quantitative differences between saliva from sNP and $\mathrm{sP}$ rats were found for some SMR2 fragments and RSP1, the latter being more concentrated in saliva of sNP than sP rats. Conversely, the novel SMCG naturally occurring fragments characterized in this study showed a significant higher abundance in $\mathrm{SP}$ than sNPs rats. SMR1, gliadoralin, and SMGM protein species, such as several uncharacterized components, showed similar levels in the two rat populations.

Importantly, the two groups of rats studied had not been exposed to alcohol before the experiments; thus, their SM protein profiles were genetically determined. Rats of the sP and sNP lines have been selectively bred for opposite alcohol preference and consumption for 85 generations. Based on the high number of generations produced to date and limited genetic combination (relatively few breeding families have indeed been available), sP and sNP rat lines can presently be considered as being practically inbred. ${ }^{1}$ Selective breeding of $\mathrm{sP}$ and SNP rats has resulted in several traits differing between the two rat lines; for example, $\mathrm{sP}$ rats display several anxiety-related behaviors when exposed to an anxiogenic environment. ${ }^{1}$ It is not clear, however, if these differential traits are associated with alcohol preference and avoidance or are simply the result of a 
genetic drift. This picture likely applies also to the qualitative and quantitative differences in the protein profile of SM saliva. Accordingly, it would be of interest to investigate whether the variations in salivary proteomics observed in $\mathrm{sP}$ and $\mathrm{sNP}$ rats may generalize to other lines of rats selectively bred for opposite alcohol preference and consumption. An extension of the differences observed in $\mathrm{sP}$ and $\mathrm{sNP}$ rats to other rat lines, e.g., Indiana $\mathrm{P}$ and $\mathrm{NP}$ rats, $\mathrm{HAD}$ and $\mathrm{LAD}$ rats, and Alko Alcohol and Alko Non-Alcohol rats, would strengthen the hypothesis of the existence of a correlation between salivary proteomics and alcohol preference and consumption. Additionally, the results of the present study prompt us to investigate if and how chronic, voluntary alcohol drinking in $\mathrm{sP}$ rats may alter salivary proteome and alcohol-taste reactivity.

\section{ASSOCIATED CONTENT}

\section{S Supporting Information}

The Supporting Information is available free of charge on the ACS Publications website at DOI: 10.1021/acs.jproteome.7b00632.

Figures showing the sequences of SMGC, SMR2, CRP, GRP-B, GRP-CB, Gliadoralin A, SMGM, and SMR1, and the cleavage sites generating naturally occurring peptides. Tables showing Mav values, elution times, and multiply charged ions selected for XIC quantification of proteins and peptides identified and detectable in HPLC-lowresolution-ESI-MS with a frequency $\geq 40 \%$; and mass values, elution times, and multiply charged ions selected for XIC quantification of peptides and proteins not identified. (PDF)

\section{AUTHOR INFORMATION}

\section{Corresponding Author}

*E-mail: tcabras@unica.it. Phone: +39-070-6754500. Fax: +39070-6754523.

\section{ORCID $\odot$}

Tiziana Cabras: 0000-0001-7535-9825

\section{Notes}

The authors declare no competing financial interest.

Mass spectrometry data are available free of charge on the ProteomeXchange Consortium via the PRIDE partner repository with the data set identifier PXD006997 (https:// www.ebi.ac.uk/pride/archive/).

\section{ACKNOWLEDGMENTS}

J.E. acknowledges support from Regione Autonoma della Sardegna within the frame of the Visiting Professor program at the University of Cagliari for appointment as Visiting Professor (Call July 2014-15), joint invitation by the Dipartimento di Scienze Biomediche e di Scienze della Vita e dell'Ambiente, Università di Cagliari, 09042 Monserrato (Cagliari), Italy). This work was supported by Cagliari University (CAR_2014), Catholic University of Rome (D1R4124500372), and National Research Council of Italy (CNR) (DSB.AD004.077). The content is solely the responsibility of the authors and does not necessarily represent the official views of the National Institutes of Health.

\section{ABBREVIATIONS}

sP, Sardinian alcohol-preferring; sNP, Sardinian alcohol-nonpreferring; SM, submandibular; BCA, bicinchoninic acid; RP, reverse-phase; XIC, extracted ion current; TIC, total ion current; Mav, average mass; Monois, monoisotopic; M, mass; ET, elution time; SMGC, submandibular gland protein C; SMR2, submandibular rat protein 2; Pyro-Glu, pyro-glutamic acid; RSP1, rat salivary protein 1; GRP, glutamine and glutamic acid rich protein; A, gliadoralin A; SMGM, submandibular gland mucin; SMR1, submandibular rat protein 1

\section{REFERENCES}

(1) Colombo, G.; Lobina, C.; Carai, M. A. M.; Gessa, G. L. Phenotypic characterization of genetically selected Sardinian alcoholpreferring (sP) and -non preferring (sNP) rats. Addict. Biol. 2006, 11, 324-338.

(2) Loi, B.; Lobina, C.; Maccioni, P.; Fantini, N.; Carai, M. A. M.; Gessa, G. L.; Colombo, G. Increase in alcohol intake, reduced flexibility of alcohol drinking, and evidence of signs of alcohol intoxication in Sardinian alcohol-preferring rats exposed to intermittent access to 20\% alcohol. Alcohol.: Clin. Exp. Res. 2010, 34, 21472154.

(3) Lobina, C.; Agabio, R.; Diaz, G.; Fà, M.; Fadda, F.; Gessa, G. L.; Reali, R.; Colombo, G. Constant absolute ethanol intake by alcoholpreferring sP rats independent of ethanol concentrations. Alcohol Alcohol. 1997, 32, 19-22.

(4) Agablo, R.; Caral, M. A. M.; Lobina, C.; Pani, M.; Reali, R.; Bourov, I.; Gessa, G. L.; Colombo, G. Dissociation of alcohol and saccharin preference in sP and SNP rats. Alcohol.: Clin. Exp. Res. 2000, 24, 24-29.

(5) Bice, P. J.; Kiefer, S. W. Taste reactivity in alcohol preferring and nonpreferring rats. Alcohol.: Clin. Exp. Res. 1990, 14, 721-727.

(6) Fábián, T. K.; Beck, A.; Fejérdy, P.; Hermann, P.; Fábián, G. Molecular Mechanisms of Taste Recognition: Considerations about the Role of Saliva. Int. J. Mol. Sci. 2015, 16, 5945-5974.

(7) Cabras, T.; Melis, M.; Castagnola, M.; Padiglia, A.; Tepper, B. J.; Messana, I.; Barbarossa, I. T. Responsiveness to 6-n-Propylthiouracil (PROP) Is Associated with Salivary Levels of Two Specific Basic Proline-Rich Proteins in Humans. PLoS One 2012, 7, e30962.

(8) Ekström, J.; Murakami, M.; Inzitari, R.; Khosravani, N.; Fanali, C.; Cabras, T.; Fujita-Yoshigaki, J.; Sugiya, H.; Messana, I.; Castagnola, M. RP-HPLC-ESI-MS characterization of novel peptide fragments related to rat parotid secretory protein in parasympathetic induced saliva. J. Sep. Sci. 2009, 32, 2944-2952.

(9) Cabras, T.; Iavarone, F.; Pirolli, D.; De Rosa, M. C.; Vitali, A.; Faa, G.; Cordaro, M.; Messana, I.; Ekstrom, J.; Castagnola, M. Topdown HPLC-ESI-MS characterization of rat gliadoralin A, a new member of the family of rat submandibular gland glutamine-rich proteins and potential substrate of transglutaminase. J. Sep. Sci. 2013, $36,2848-2861$.

(10) Alves, R. M. P.; Vitorino, R.; Padrao, A. I.; Moreira-Goncalves, D.; Duarte, J. A.; Ferreira, R. M. P.; Amado, F. iTRAQ-based quantitative proteomics analysis of submandibular glands from rats with STZ-induced Hyperglycemia. J. Biochem. 2013, 153, 209-220.

(11) Alterman, A.; Mathison, R.; Coronel, C. E.; Stroppa, M. M.; Finkelberg, A. B.; Gallarà, R. V. Functional and proteomics analysis of submandibular saliva in rats exposed to chronic stress by immobilization or constant light. Arch. Oral Biol. 2012, 57, 663-669.

(12) Zhang, Z.; Marshall, A. G. An universal algorithm for fast and automated charge state deconvolution of electrospray mass-to-charge ratio spectra. J. Am. Soc. Mass Spectrom. 1998, 9, 225-233.

(13) Vizcaíno, J. A.; Csordas, A.; Del-Toro, N.; Dianes, J. A.; Griss, J.; Lavidas, I.; Mayer, G.; Perez-Riverol, Y.; Reisinger, F.; Ternent, T.; Xu, Q. W.; Wang, R.; Hermjakob, H. 2016 update of the PRIDE database and related tools. Nucleic Acids Res. 2016, 44 (44), D447-D456.

(14) Ong, S. E.; Mann, M. Mass spectrometry-based proteomics turns quantitative. Nat. Chem. Biol. 2005, 1, 252-262. 
(15) Messana, I.; Inzitari, R.; Fanali, C.; Cabras, T.; Castagnola, M. Facts and artifacts in proteomics of body fluids. What proteomics of saliva is telling us? J. Sep. Sci. 2008, 31, 1948-1963.

(16) Vizcaíno, J. A.; Deutsch, E. W.; Wang, R.; Csordas, A.; Reisinger, F.; Ríos, D.; Dianes, J. A.; Sun, Z.; Farrah, T.; Bandeira, N.; Binz, P. A.; Xenarios, I.; Eisenacher, M.; Mayer, G.; Gatto, L.; Campos, A.; Chalkley, R. J.; Kraus, H. J.; Albar, J. P.; Martinez-Bartolomé, S.; Apweiler, R.; Omenn, G. S.; Martens, L.; Jones, A. R.; Hermjakob, H. ProteomeXchange provides globally co-ordinated proteomics data submission and dissemination. Nat. Biotechnol. 2014, 32, 223-226.

(17) Zinzen, K. M.; Hand, A. R.; Yankova, M.; Ball, W. D.; Mirels, L. Molecular cloning and characterization of the neonatal rat and mouse submandibular gland protein SMGC. Gene 2004, 334, 23-33.

(18) Denny, P. C.; Mirels, L.; Denny, P. A. Mouse submandibular gland salivary apomucin contains repeated $\mathrm{N}$-glycosylation sites. Glycobiology 1996, 6, 43-50.

(19) Rougeot, C.; Rosinski-Chupin, I.; Njamkepo, E.; Rougeon, F. Selective processing of submandibular rat 1 protein at dibasic cleavage sites. Eur. J. Biochem. 1994, 219, 765-773.

(20) Ekström, J.; Månsson, B.; Tobin, G. Vasoactive intestinal peptide evoked secretion of fluid and protein from rat salivary glands and the development of supersensitivity. Acta Physiol. Scand. 1983, $119,169-175$

(21) Ohlin, P. Isoprenaline as a secretory agent in salivary glands. Acta Universitatis Lundensis. Sectio II 1964, 17, 1-8.

(22) Messana, I.; Cabras, T.; Pisano, E.; Sanna, M. T.; Olianas, A.; Manconi, B.; Pellegrini, M.; Paludetti, G.; Scarano, E.; Fiorita, A.; Agostino, S.; Contucci, A. M.; Calò, L.; Picciotti, P. M.; Manni, A.; Bennick, A.; Vitali, A.; Fanali, C.; Inzitari, R.; Castagnola, M. Trafficking and postsecretory events responsible for the formation of secreted human salivary peptides: a proteomics approach. Mol. Cell. Proteomics 2008, 7, 911-926.

(23) Van Coillie, E.; Proost, P.; Van Aelst, I.; Struyf, S.; Polfliet, M.; De Meester, I.; Harvey, D. J.; Van Damme, J.; Opdenakker, G. Functional comparison of two human monocyte chemotactic protein-2 isoforms, role of the amino-terminal pyroglutamic acid and processing by CD26/dipeptidyl peptidase IV. Biochemistry 1998, 37, 1267212680.

(24) Goren, H. J.; Bauce, L. G.; Vale, W. Forces and structural limitations of binding of thyrotrophin-releasing factor to the thyrotrophin-releasing receptor: the pyroglutamic acid moiety. Mol. Pharmacol. 1977, 13, 606-614.

(25) Rosinski-Chupin, I.; Rougeot, C.; Courty, Y.; Rougeon, F. Localization of mRNAs of two androgen-dependent proteins, SMR1 and SMR2, by in situ hybridization reveals sexual differences in acinar cells of rat submandibular gland. J. Histochem. Cytochem. 1993, 41, 1645-1649.

(26) Dennison, S. R.; Mura, M.; Harris, F.; Morton, L. H.; Zvelindovsky, A.; Phoenix, D. A. The role of C-terminal amidation in the membrane interactions of the anionic antimicrobial peptide, maximin H5. Biochim. Biophys. Acta, Biomembr. 2015, 1848, 11111118

(27) Rosinski-Chupin, I.; Rougeon, F. The gene encoding SMR1, a precursor-like polypeptide of the male rat submaxillary gland, has the same organization as the preprothyrotropin-releasing hormone gene. DNA Cell Biol. 1990, 9, 553-559.

(28) Rougeot, C.; Rosinski-Chupin, I.; Njamkepo, E.; Rougeon, F. Selective processing of submandibular rat 1 protein at dibasic cleavage sites. Salivary and bloodstream secretion products. Eur. J. Biochem. 1994, 219, 765-773.

(29) Rougeot, C.; Messaoudi, M.; Hermitte, V.; Rigault, A. G.; Blisnick, T.; Dugave, C.; Desor, D.; Rougeon, F. Sialorphin, a natural inhibitor of rat membrane-bound neutral endopeptidase that displays analgesic activity. Proc. Natl. Acad. Sci. U. S. A. 2003, 100, 8549-8554.

(30) Morris, K.; Kuo, B.; Wilkinson, M. D.; Davison, J. S.; Befus, A. D.; Mathison, R. D. Vcsal gene peptides for the treatment of inflammatory and allergic reactions. Recent Pat. Inflammation Allergy Drug Discovery 2007, 1, 124-132.
(31) Mathison, R. D.; Davison, J. S.; Saint Laurent, C. D.; Befus, A. D. Autonomic regulation of anti-inflammatory activities from salivary glands. Chem. Immunol. Allergy 2012, 98, 176-195.

(32) Emmelin, N.; Holmberg, J.; Ohlin, P. Receptors for catecholamines in the submaxillary glands of rats. Br. J. Pharmacol. Chemother. 1965, 25, 134-138.

(33) Mirels, L.; Bedi, G. S.; Dickinson, D. P.; Gross, K. W.; Tabak, L. A. Molecular characterization of glutamic acid/glutamine-rich secretory proteins from rat submandibular glands. J. Biol. Chem. 1987, 262, 7289-7297.

(34) Cooper, L. F.; Elia, D. M.; Tabak, L. A. Secretagogue-coupled Changes in the Expression of Glutamine/Glutamic Acid-rich Proteins (GRPs) Isoproterenol induces changes in GRP transcript expression and changes in isoforms secreted. J. Biol. Chem. 1991, 266, 35323539.

(35) Chao, J. Rat kallikrein 2, tonin. In Handbook of Proteolytic Enzymes, 2nd ed.; Barrett, A. J.; Rawlings, N. D.; Woessner, J. F., Ed.; Elsevier: London, 2004; pp 1608-1610.

(36) Moreau, T.; Brillard-Bourdet, M.; Bouhnik, J.; Gauthier, F. Protein products of the rat kallikrein gene family. Substrate specificities of kallikrein rK2 (tonin) and kallikrein rK9. J. Biol. Chem. 1992, 267, $10045-10051$. 